\title{
Photoelectron spectroscopy of ethylene, isobutylene, trimethylethylene, and tetramethylethylene at variable angle $e^{a)}$
}

\author{
Donald M. Mintz ${ }^{\text {b) }}$ and Aron Kuppermann
}

Arthur Amos Noyes Laboratory of Chemical Physics, c) California Institute of Technology, Pasadena, California 91125

(Received 13 November 1978)

\begin{abstract}
Using a Hel line $58.4 \mathrm{~nm}$ source lamp, photoelectron angular distributions were measured for the series of four olefins: ethylene, isobutylene (2-methyl-propene), trimethylethylene (2-methyl-2-butene), and tetramethylethylene (2,3-dimethyl-2-butene). From these, the asymmetry parameter $\beta$ as a function of photoelectron energy was obtained for each of these molecules. The following important effects in the behavior of $\beta$ are observed: (a) In the $\pi$ orbital ionization regions of the spectrum of each molecule, $\beta$ increases with increasing electron energy across the vibrational envelopes. (b) With increasing methyl substitution (and at a fixed photoelectron enegy) $\beta$ for this band decreases. (c) In the region of the spectra of each of the methyl-substituted ethylenes involving several $2 p \sigma$ bands, this energy dependence of $\beta$ behaves as if they constituted a single band, in spite of the widely differing orbital symmetries. (d) Over most of the $2 p \sigma$ region of each molecule, $\beta$ decreases with increasing photoelectron energy, except for the high ionization potential end of this region, where $\beta$ increases instead. We attribute effects (b) and (d) to $\sigma-\pi$ orbital mixing.
\end{abstract}

\section{INTRODUCTION}

The assignment of the structure observed in the vacuum ultraviolet photoelectron spectra of polyatomic molecules is complicated by the high density of ionic electronic states in the ionization potential range 8-20 eV. Nevertheless, in the orbital approximation, ${ }^{1}$ each band in a molecular photoelectron spectrum corresponds to ionization from a particular molecular orbital.

Several experimental approaches have been useful for the assignment of structure in photoelectron spectra. These include analysis of vibrational fine structure, ${ }^{1,2}$ comparison of photoelectron spectra of related compounds, ${ }^{3-5}$ and the observation of the variation of band intensities with photon energy ${ }^{6-9}$ Analysis of vibrational structure is normally only possible for simple molecules. For polyatomic molecules, Franck-Condon factors linking the ground neutral states to many different modes of vibration of the ions are frequently important. The high density of vibrational states of such polyatomic ions makes their resolution in photoelectron spectroscopy very difficult. Commonly studied series of related molecules involve varying degrees of substitution of hy drogen atoms by bulky alkyl groups or by highly electronegative fluorines. The resulting substituent effects must be interpreted with caution, ${ }^{5}$ since it is easy for those substituents to grossly perturb geometries and electronic densities. The study of band intensity variation with photon energy has firm theoretical grounds only in the energy range well above $100 \mathrm{eV},{ }^{8}$ though it has been applied for comparisons of He I and He II spectra." ${ }^{7,8}$ For polyatomic molecules, these several ap-

a) This work was supported in part by a contract (EY-76-S-03767) from the Department of Energy. Report Code: CALT767 P4-178.

b) Work performed in partial fulfillment of the requirements for the Ph. D. degree in Chemistry at the California Institute of Technology. Present address: Finnigan Corporation, Sunnyvale, CA 94086 .

c) Contribution No. 5884 . proaches are frequently insufficient for making reliable photoelectron band assignments.

In the past ten years, a new and promising experimental technique has been developed and used as an aid in such assignments: the measurements of photoelectron angular distributions and of the corresponding anisotropy parameters, $\beta$. In 1968, Hall and Siegel ${ }^{10}$ found that ejected electron angular distributions in the laser photodetachment of $\mathrm{H}^{-}$and $\mathrm{O}^{-}$were very much different. Their data were theoretically attributed by Cooper and Zare $^{11}$ to the $s$ character in the $\mathrm{H}^{*}$ electrons and the $p$ character of the outer shell $\mathrm{O}^{-}$electrons.

A similar dependence of the anisotropy parameter on orbital angular momentum has been found for the photoionization of polyatomic molecules. It is expected that this parameter will also depend on other characteristics of the molecule's electronic structure. Since their calculation presents great practical difficulties, it is appropriate to find the factors influencing $\beta$ by using an empirical approach. If empirical rules governing the behavior of $\beta$ can be found, they can be used to help assign the structure in photoelectron spectra. Such assignments are useful for the assessment of different molecular quantum mechanical calculations of the energies of electronic states of ions. Different calculations sometimes result in conflicting assignments of the energy ordering of those states.

Carlson et al..$^{12,13}$ have compared angular distributions of molecular photoelectrons arising from a given molecular orbital to values of the most probable angular momentum in a single center expansion of that orbital. As Rabalais et al. ${ }^{14}$ have pointed out, this correlation can be theoretically justified only for ionizations yielding photoelectrons of high energies. To date, most experimental angular distribution studies of molecules have focused on the ionization of valence electrons within 15 $\mathrm{eV}$ of threshold, and do not satisfy this condition. It is appropriate to search for additional kinds of correla- 


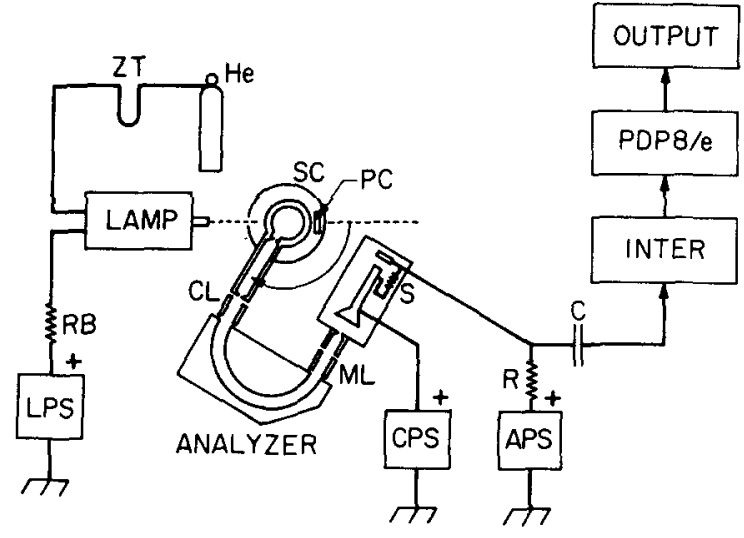

FIG. 1. Block diagram of variable angle photoelectron spectrometer. He, cylinder of UHP (ultra high purity) helium; $\mathrm{ZT}$, liquid nitrogen immersed zeolite trap for lamp helium supply; BR, lamp ballast resistor; LPS, lamp de power supply; SC, sample chamber; $\mathrm{PC}$, photocathode for light flux measurements; CL and ML, electron lens elements; ANALYZER, hemispherical electrostatic electron energy analyzer; $S$, Spiraltron channel electron multiplier; CPS and APS, power supplies for Spiraltron channel electron cathode and anode; $\mathrm{R}, \mathrm{C}$, differentiating network for Spiraltron pulses; PDP8/e, Digital Equipment Corporation PDP8/e minicomputer; INTER, counting system interface to experiment; OUT PUT, computer output devices to user.

tions between the behavior of $\beta$ and electronic structure properties.

In this spirit, we present in the present paper the results of a series of experimental measurements of photoelectron angular distributions of ethylene, isobutylene (2-methyl-propene), trimethylethylene (2-methyl-2butene), and tetramethylethylene (2,3-dimethyl-2-bu-

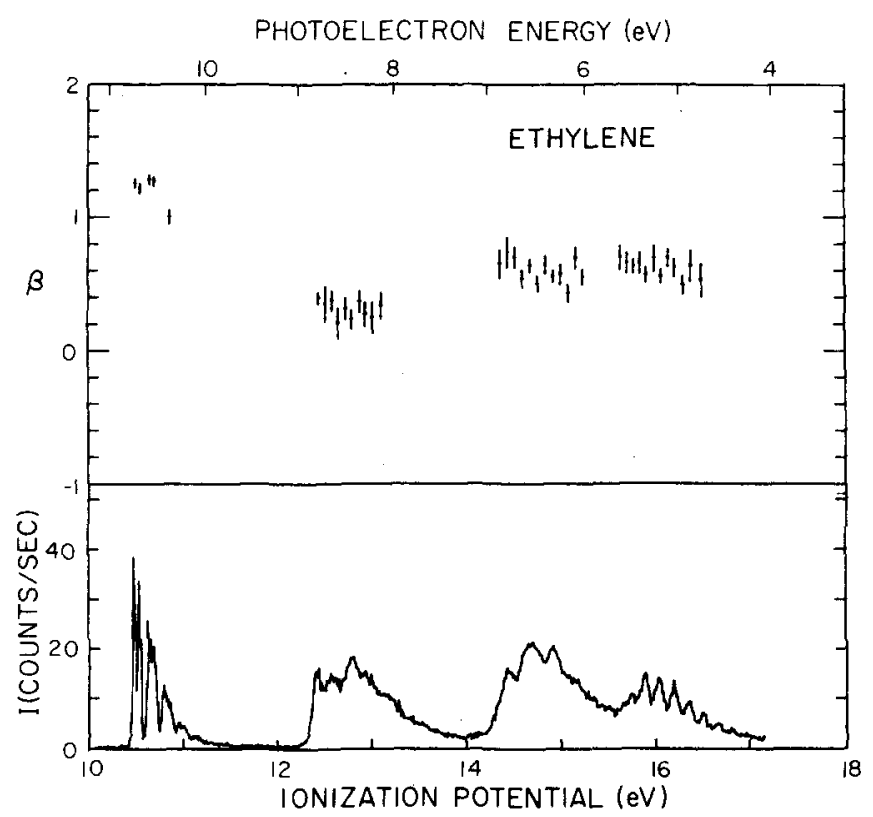

FIG. 2. Photoelectron spectrum (lower panel) and variation of $\beta$ with ionization potential and photoelectron energy (upper panel) for ethylene using $58.4 \mathrm{~nm}(21.22 \mathrm{eV})$ radiation. The spectrum was obtained at a detector angle of $54.7^{\circ}$ using adjacent $14 \mathrm{meV}$ wide channels and 50 scans. Total dwell time per channel was $50 \mathrm{sec}$.

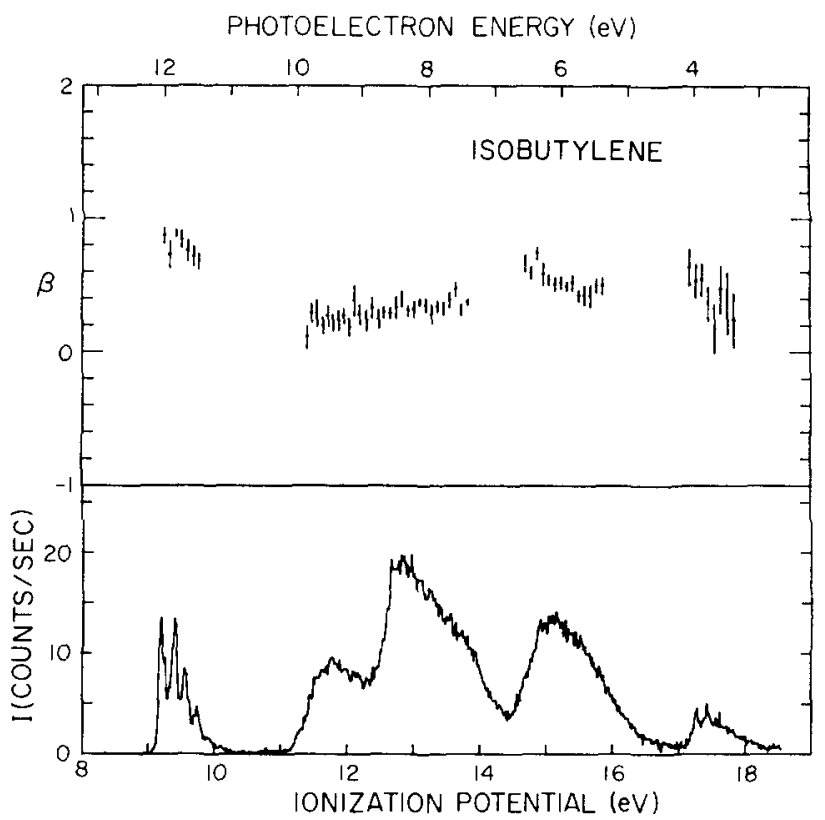

FIG. 3. Photoelectron spectrum (lower panel) and variation of $\beta$ with ionization potential and photoelectron energy (upper pane1) for isobutylene using $58.4 \mathrm{~nm}(21.22 \mathrm{eV})$ radiation. The spectrum was obtained at a detector angle of $54.7^{\circ}$ using adjacent $20 \mathrm{meV}$ wide channels and 60 scans. Total dwell time per channel was $60 \mathrm{sec}$.

tene). Very early preliminary work on ethylene ${ }^{15}$ is hereby superseded. Carlson and co-workers ${ }^{16}$ have made analogous measurements on ethylene, propylene, and $c i s$ - and trans-2-butene. These two series of molecules jointly provide $\beta$ parameters for ethylene and all of its methyl-substituted derivatives. This permits a detailed examination of the effect of methyl substitution in this series of related molecules.

\section{EXPERIMENTAL}

The experimental apparatus has been described in detail previously ${ }^{17}$ and is summarized here only briefly. It consists of a windowless helium glow discharge lamp (mainly $58.4 \mathrm{~nm}$ ) and a rotatable electron energy analyzer. The latter incorporates a $6.350 \mathrm{~cm}$ mean radius hemispherical electrostatic analyzer having a $1.270 \mathrm{~cm}$ radial gap, ${ }^{17}$ electrostatic lenses, and an electron multiplier detector. The entire analyzer is mounted on a worm gear which is rotated about a horizontal axis, and is attached to a sample chamber which is normally filled with 3-5 mTorr of the sample gas under study. The photoelectron intensity is measured as a function of electron energy using a minicomputer-based data acquisition system. A block diagram of the apparatus is given in Fig. 1.

Sample materials were obtained from the following sources and at the manufacturer stated minimum purities indicated in parentheses: ethylene (J. T. Baker, CP 99. 5\%), isobutylene (Matheson, CP 99\%), 2-methyl2-butene (trimethylethylene) (Aldrich, 99+\%), and 2,3dimethyl-2-butene (tetramethylethylene) (Aldrich, Gold Label $99+\%$ ). Full spectra of these compounds were taken at the magic detector angle of $54.7^{\circ}$ with respect to the light axis and are reproduced in Figs. 2-5. The 


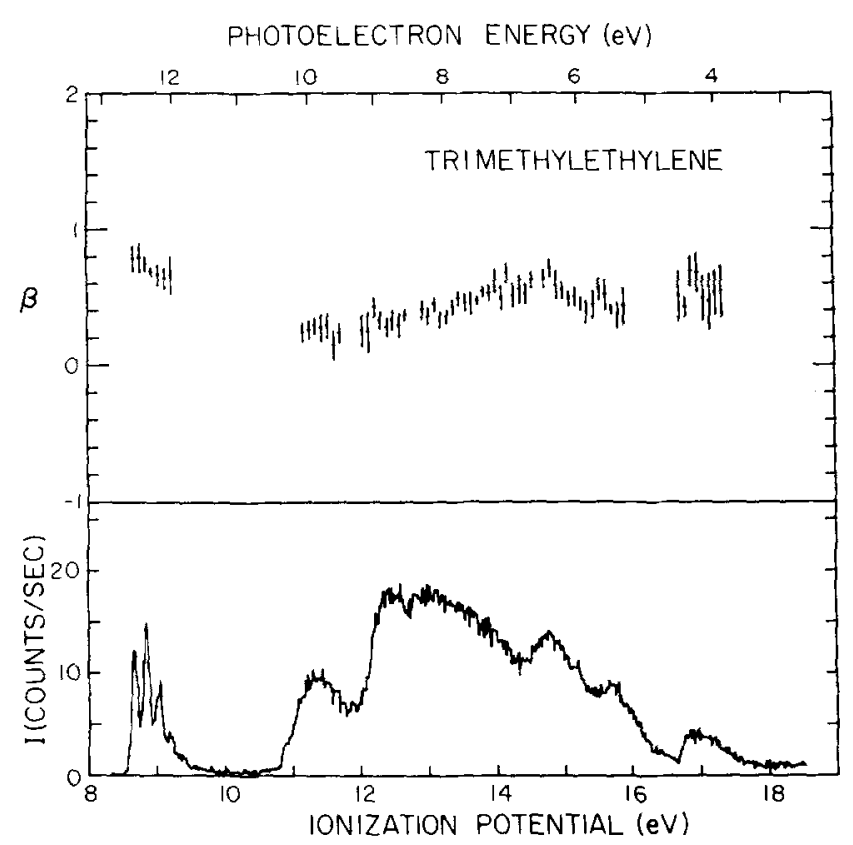

FIG. 4. Photoelectron spectrum (lower panel) and variation of $\beta$ with ionization potential and photoelectron energy (upper panel) for trimethylethylene using $58.4 \mathrm{~nm}(21.22 \mathrm{eV})$ radiation. The spectrum was obtained at a detector angle of $54.7^{\circ}$ using adjacent $20 \mathrm{meV}$ wide channels and 60 scans. Total dwell time per channel was $60 \mathrm{sec}$.

spectra of ethylene and isobutylene display the same structure as previously published high resolution photoelectron spectra. ${ }^{18-20}$

Liquid samples were degassed by several freezepump-thaw cycles before use. Gas samples were distilled from the gas cylinder into a liquid nitrogen-immersed cold finger and were further degassed by several freeze-pump-thaw cycles. The glass manifold which constituted the inlet system was baked in a glassblower's annealing oven between successive samples in order to avoid cross contamination.

Spectra were taken at an analyzing energy of $1.5 \mathrm{eV}$. The corresponding resolution, as measured at the ${ }^{2} P_{3 / 2}$ peak of argon, was $30-35 \mathrm{meV}$ full width at half maximum (FWHM). The electron energy scale was calibrated with the argon ${ }^{2} P_{3 / 2}$ peak at $15.759 \mathrm{eV}$ ionization potential. Furthermore, the linearity of the energy scale was verified with the help of a spectrum of a mixture containing approximately equal amounts of ethylene, xenon, and argon.

Total data acquisition times averaged about $100 \mathrm{~h}$ per substance. This included the time required for obtaining a full $54.7^{\circ}$. spectrum, angular distributions covering detector angles of $40^{\circ}-120^{\circ}$ in increments of $10^{\circ}$, and a background spectrum at each of these nine angles. Each spectrum in the angular distribution consisted of intensity measurements which scanned an interval of electron kinetic energies of width 0.5 to $1.5 \mathrm{eV}$ using 80-160 channels for data storage. During that period of four days, the lamp flux, as measured with a tungsten photocathode mounted in the sample chamber, did not vary by more than $5 \%$. Over shorter periods of $6-10 \mathrm{~h}$ during which an angular distribution was run, the lamp flux typically did not vary by more than $1 \%$. The positions of sharp features in the spectrum did not change by more than $0.01 \mathrm{eV}$ over $6-10 \mathrm{~h}$.

Spectral intensities at each of the nine angles and at each channel of the spectrum were then fitted to the theoretical expression ${ }^{21}$

$$
I(\theta)=\alpha \frac{Q}{4 \pi}\left[1-\frac{\beta}{2} P_{2}(\cos \theta)\right],
$$

where $\theta$ is the angle between the directions of the incident (unpolarized) light beam and of the ejected photoelectron, $I(\theta)$ is the photoelectron intensity corrected for the volume of intersection of lamp and detector view cones at each $\theta$ and compensated for the linear variation of counting rate with sample pressure, $Q$ is the integral photoionization cross section, $\alpha$ is an instrumental sensitivity constant, and $\beta$ is the fitted asymmetry parameter. The latter must lie in the range of -1 to +2 . The photoelectron intensity is also corrected for an energy parameterized background which is different at each detector angle. Photoelectron angular distributions were measured over at least a $10 \mathrm{eV}$ range starting below the first adiabatic ionization potential (I.P.). Intensities at electron energies corresponding to ionization potentials above $19 \mathrm{eV}$ were in general too low for the molecules used in this study to yield adequate signal to background ratios.

\section{RESULTS}

\section{A. Ethylene}

The low resolution $58.4 \mathrm{~nm}$ photoelectron spectrum of ethylene was first published in $1964 . .^{22}$ Since that

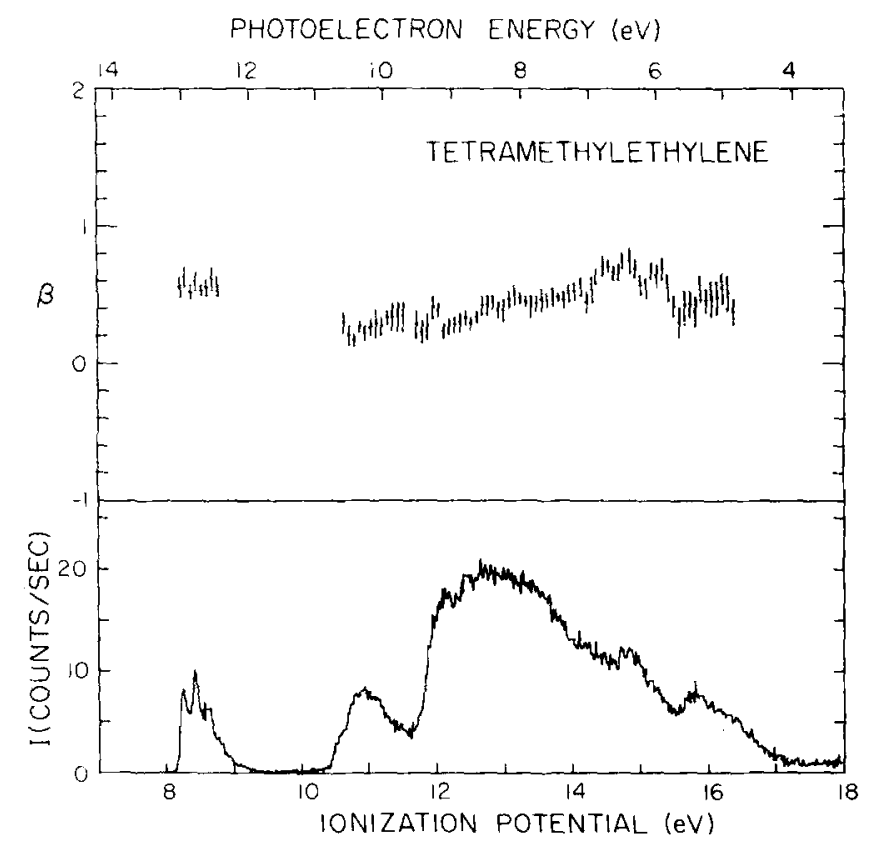

FIG. 5. Photoelectron spectrum (lower panel) and variation of $\beta$ with ionization potential and photoelectron energy (upper panel) for tetramethylethylene using $58.4 \mathrm{~nm}(21.22 \mathrm{eV})$ radiation. The spectrum was obtained at a detector angle of $54.7^{\circ}$ using adjacent $20 \mathrm{meV}$ wide channels and 60 scans. Total dwell time per channel was 60 sec. 
TABLE I. Characteristics of the photoelectron spectra of ethylene.

\begin{tabular}{|c|c|c|c|c|c|}
\hline \multirow[b]{2}{*}{$\begin{array}{l}\text { Vertical } \\
\text { I. P. (eV) }\end{array}$} & \multirow[b]{2}{*}{$\begin{array}{c}\text { Orbital } \\
\text { symmetry }\end{array}$} & \multirow[b]{2}{*}{$\begin{array}{c}\text { Bonding } \\
\text { character }^{\mathrm{b}, \mathrm{c}}\end{array}$} & \multicolumn{3}{|c|}{ Vertical $^{\ominus}$} \\
\hline & & & Range across bands ${ }^{d}$ & This work & $\begin{array}{l}\text { Previous } \\
\text { work }^{c}\end{array}$ \\
\hline 10.51 & $1 b_{3 u}$ & $\pi$ & $1.00 \pm 0.10-1.25 \pm 0.05$ & $1.25+0.05$ & 1.20 \\
\hline 12.82 & $1 b_{3 g}$ & $\begin{array}{l}\mathrm{C} 2 p \sigma, \text { principally } \\
\mathrm{CH} \text { bonding }\end{array}$ & $0.23 \pm 0.10-0.40 \pm 0.09$ & $0.30 \pm 0.05$ & 0.35 \\
\hline 14.69 & $3 a_{8}$ & $\begin{array}{l}\mathrm{C} 2 p \sigma, \text { principally } \\
\mathrm{CC} \text { bonding }\end{array}$ & $0.48 \pm 0.05-0.73 \pm 0.07$ & $0.60 \pm 0.10$ & 0.60 \\
\hline 15.90 & $1 b_{2 u}$ & $\begin{array}{l}\mathrm{C} 2 p \sigma, \text { principally } \\
\mathrm{CH} \text { bonding }\end{array}$ & $0.41 \pm 0.15-0.75 \pm 0.08$ & $0.65 \pm 0.05$ & 0.65 \\
\hline 19.1 & $2 b_{1 u}$ & $\begin{array}{l}\mathrm{C} 2 s \sigma, \text { slightly } \mathrm{CC} \\
\text { antibonding }\end{array}$ & & & \\
\hline
\end{tabular}

arbital designation from Ref. 18a. The overall symmetry of ethylene is $D_{2 \mathrm{k}}$.

beference 18 .

'Reference 16 .

The first and second values given are, respectively, the lowest and highest values of $\beta$ for the band. The uncertainties are obtained from the goodness-of-fit of the data to Eq. (1) (see Ref. 17).

${ }^{\circ}$ Evaluated at vertical ionization potential.

time, several groups have obtained high resolution spectra of this molecule. ${ }^{18,23}$ They consist of five vibrationally structured bands, the first four of which are displayed in Fig. 2. The first band, at a vertical ionization potential of $10.51 \mathrm{eV}$, corresponds to the loss of a carbon-carbon bonding $\pi$ electron, as is typical of unsaturated hydrocarbons. 24 Features at vertical I. P.'s of 12. 82, 14,69, and $15.90 \mathrm{eV}$ correspond to the loss of $\sigma$ orbital electrons. All three of these $\sigma$ bands correspond to the loss of electrons which are at least partially $\mathrm{CH}$ bonding, as indicated by analyses of their vibrational structure..$^{18,23}$ However, the one at $14.69 \mathrm{eV}$ is due to ionization from a predominantly $\mathrm{C}-\mathrm{C}$ bonding orbital. The fifth band appears near $19 \mathrm{eV}$ and is extremely weak. ${ }^{18}$ As a result, we did not attempt to obtain angular distributions for it.

Table I contains the I. P.'s, the symmetry, and bonding characteristics of the orbitals from which ionization occurs, and our values of $\beta$ as well as those of White $e t$ al..$^{16}$ As previously observed, ${ }^{13,15,16}$ the angular distribution for the removal of the ethylene $\pi$ electron is noticeably different from those for the removal of the $\sigma$ electrons. The $\beta$ value corresponding to the vertical ionization of the $\pi$ electron is large, $1.25 \pm 0.05$. The measured $\beta$ values in the $\sigma$ region are very much lower and lie in the range of $0.30-0.65$.

\section{B. Isobutylene}

The high resolution $58.4 \mathrm{~nm}$ photoelectron spectrum of isobutylene has been published previously only by Kimura et al..$^{10}$ and by Wiberg et al..$^{20}$ and consists of five distinct bands and is shown in Fig. 3. The only bands which display vibrational structure are those at vertical ionization potentials of 9.45 and $17.27 \mathrm{eV}$. The former corresponds to ionization of a $\pi$ electron and has a $C=C$ stretching frequency of $(1400 \pm 50) \mathrm{cm}^{-1}$. The latter corresponds to the removal of an electron from an orbital with a large amount of $2 s$ character and shows a $\mathrm{CH}_{n}$ deformation frequency $\mathrm{y}^{25(2)}$ of $(1350 \pm 50) \mathrm{cm}^{-1}$.

Kimura and collaborators ${ }^{18}$ have performed equivalent orbital $^{28}$ calculations for isobutylene. From their results, their spectrum, and the sum rules inherent in that method of calculation, they identify seven $C 2 p \sigma$ bands in the range of ionization potentials $11-17 \mathrm{eV}$. These have varying degrees of $\mathrm{CH}$ and $\mathrm{CC}$ bonding character. Wiberg et al. ${ }^{20}$ further assign the in-plane C2po structure on the basis of an ab initio calculation. Their results disagree with those of Kumura et al. ${ }^{19}$ on the energy ordering of the $7 a_{1}, 3 b_{2}$, and $1 b_{1}$ bands. The reliability of the sum rules and the inherent error of the scaled $a b$ initio results $(\sim 0.22 \mathrm{eV})$ support the Kimura suggestion that the $1 b_{1}$ ionization (out of plane) lies at $\sim 15.7 \mathrm{eV}$, which falls in the high I.P. end of the C2po region. Table II contains selected ionization potentials (some of which are identified by Kimura et al. ${ }^{19(2)}$ ), the characteristics of the orbitals from which ionization occurs, and our values of $\beta$. The value of this parameter for the $\pi$ ionization $(0.75)$ is noticeably higher than that for the $\sigma$ electron $(0.25)$ at an I.P. of $11.81 \mathrm{eV}$.

In Fig. 6 we plot $\beta$ against photoelectron kinetic energy $E_{0}$ for the 2po I. P.'s listed by Kimura et al. ${ }^{19(2)}$ Most of the points fall on a straight line, but the one at the lowest energy, (corresponding to the shoulder in the spectrum of Fig. 3 at $15.7 \mathrm{eV}$ ) is significantly below it. An interpretation of this behavior is given in Sec. IVC.

\section{Trimethylethylene}

The high resolution photoelectron spectrum of trimethylethylene is given in Fig. 4. The only previous photoelectron study of this molecule was published by Frost and Sandhu ${ }^{27}$ and Bieri et al. ${ }^{28}$ Frost and Sandhu derived adiabatic and vertical I. P.'s from low resolution retardation analysis spectra. Bieri et al. make assign- 
TABLE II. Characteristics of the photoelectron spectra of isobutylene.

\begin{tabular}{|c|c|c|c|c|c|}
\hline \multicolumn{2}{|c|}{ Vertical I.P. (eV) } & \multirow{2}{*}{$\begin{array}{c}\text { Orbital } \\
\text { symmetry }\end{array}$} & \multirow{2}{*}{$\begin{array}{c}\text { Orbital } \\
\text { description }\end{array}$} & \multirow{2}{*}{$\begin{array}{c}\text { Range of } \beta \\
\text { across band }\end{array}$} & \multirow[b]{2}{*}{ Vertical $\beta^{\mathrm{c}}$} \\
\hline Experimental & Theoretical $^{d}$ & & & & \\
\hline 9.45 & 9.38 & $2 b_{1}$ & $\pi$ & $0.86 \pm 0.05-0.70 \pm 0.05$ & $0.75 \pm 0.05$ \\
\hline 11.81 & 12.13 & $5 b_{2}$ & $\mathrm{C} 2 p \sigma$ & $0.20 \pm 0.05-0.35 \pm 0.07$ & $0.25 \pm 0.05$ \\
\hline 12.90 & $12.87 \mid$ & \multirow{2}{*}{$8 a_{1}, 4 b_{2}$} & $\int \mathrm{C} 2 p \sigma$ & $0.27 \pm 0.06-0.40 \pm 0.05$ & $0.30 \pm 0.05$ \\
\hline$(13.2)^{\ominus}$ & $13.18\}$ & & C $2 p \sigma$ & $0.30 \pm 0.06-0.42 \pm 0.04$ & $0.35 \pm 0.05$ \\
\hline$(13.8)^{\circ}$ & 13.49 & $1 a_{2}$ & $\mathrm{C} 2 p \sigma ; \pi\left(\mathrm{CH}_{3}\right)$ & $0.32 \pm 0.04-0.48 \pm 0.05$ & $0.40 \pm 0.05$ \\
\hline 15.03 & 15.06 & $7 a_{1}$ & $\mathrm{C} 2 p \sigma$ & $0.51 \pm 0.04-0.74 \pm 0.04$ & $0.60 \pm 0.05$ \\
\hline$(15.3)^{\ominus}$ & 15.15 & $3 b_{2}$ & $\mathrm{C} 2 p \sigma$ & $0.46 \pm 0.04-0.58 \pm 0.05$ & $0.55 \pm 0.05$ \\
\hline$(15.7)^{\Theta}$ & 14.64 & $1 b_{1}$ & $\mathrm{C} 2 p \sigma ; \pi\left(\mathrm{CH}_{3}\right)$ & $0.42 \pm 0.06-0.52 \pm 0.04$ & $0.45 \pm 0.05$ \\
\hline 17.27 & 17.18 & $a^{\prime}$ & $\mathrm{C} 2 s \sigma$ & $0.20 \pm 0.15-0.74 \pm 0.16$ & $0.55 \pm 0.10$ \\
\hline
\end{tabular}

${ }^{2}$ In-plane orbitals assigned using point group $C_{s}$ but including $a_{1}$ and $b_{2}$ orbitals of the $C_{2 v}$ point group.

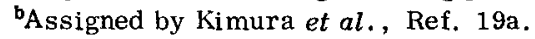

'Present work, measured at vertical ionization potential.

${ }^{\mathrm{d}}$ Reference 20 .

'Shoulders indicated in Ref. 19a.

ments only for the three bands at lowest I.P. and the C2s $\sigma$ structure lying above $16 \mathrm{eV}$. The only distinct vibrational fine structure occurs in the $\pi$ band (which has a vertical I.P. of $8.86 \mathrm{eV}$ ) and, by analogy to ethylene, corresponds to a $C=C$ stretch with frequency $(1450 \pm 50)$ $\mathrm{cm}^{-1}$. The spectrum contains a weak isolated feature with a vertical I.P. of $16.83 \mathrm{eV}$, corresponding probably to the lowest I. P. band associated with the removal of an electron with extensive carbon $2 s$ character. As jus tified in Sec. IV A, we expect to have in this molecule nine $p$-type ionizations at I.P.'s between those of the $\pi$ band and of the lowest C2so band. By analogy to ethylene, the corresponding $\mathrm{C} 2 p \sigma$ orbitals should have varying degrees of $\mathrm{CH}$ and $\mathrm{CC}$ bonding character. The feature peaking at $11.35 \mathrm{eV}$ assigned ${ }^{28}$ as $16 a^{\prime}$ seems isolated from the rest and corresponds possibly to a single band. The peak at $15.60 \mathrm{eV}$ is relatively weak and the corresponding integrated band intensity is also associated with the ionization from only one orbital. On this basis, seven overlapping ionization bands should lie in the I. P. range of about $12.0 \mathrm{eV}$ to about $15.3 \mathrm{eV}$. The lowest of these, at $12.55 \mathrm{eV}$, has been assigned ${ }^{28}$ as $15 a^{\prime}$.

In Table III we give selected I. P.'s for trimethylethylene, since the extensive band overlap in the 10.6 to 16.6 $\mathrm{eV}$ region of the spectrum makes it difficult to identify the position of all nine bands expected in that region. In addition, that table contains the characteristics of orbitals from which ionization occurs, according to the present tentative assignments, and the values of $\beta$ for the I.P.'s indicated. The $\beta$ value for the $\pi$ ionization is again higher than that of the lowest lying $2 p \sigma$ ionization measured at the vertical I.P. of $11.35 \mathrm{eV}$. Experimental $\beta$ values measured at peaks and slope breaks of the $2 p \sigma$ region between 10.6 and $16.6 \mathrm{eV} \mathrm{I.P.} \mathrm{are} \mathrm{plotted} \mathrm{as}$ a function of the corresponding electron energy in Fig. 7. The points lie on a straight line, with one exception. The $\beta$ corresponding to the peak at $15.60 \mathrm{eV} \mathrm{I.P.} \mathrm{is}$ anomalously low, and this behavior is discussed in Sec. IVC.

\section{Tetramethylethylene}

The high resolution photoelectron spectrum of tetramethylethylene is shown in Fig. 5. The only previous photoelectron investigations of this molecule were, as for the trimethylethylene, the studies of Frost and Sandhu $u^{27}$ and of Bieri et al. ${ }^{28}$ Vibrational fine structure occurs only for the $\pi$ band at $8.44 \mathrm{eV} \mathrm{I.P.} \mathrm{and} \mathrm{corre-}$ sponds to a $C=C$ stretch with frequency $(1430 \pm 50) \mathrm{cm}^{-1}$ The spectrum contains an isolated feature with maximum intensity at $10.96 \mathrm{eV} \mathrm{I.P.,} \mathrm{corresponding} \mathrm{to} \mathrm{the}$ removal of a $2 p \sigma$ electron of undetermined $\mathrm{CH}$ and $\mathrm{CC}$ character since its vibrational structure is not resolved. Bieri et $a l .{ }^{28}$ have assigned this feature as $4 b_{3 \ell}$ and the onset at $12.2 \mathrm{eV}$ as $6 a_{z}$. In addition, a shoulder at 16.4 eV I. P. probably corresponds to the removal of the least tightly bound electron having extensive carbon $2 s$ character, on the basis of comparisons to other methylated ethylenes. The other component of this high ionization potential band, with a vertical I.P. of $15.85 \mathrm{eV}$, probably results from the ionization of a single C2po electron. Since we must in this molecule account for $11 \mathrm{C} 2 p \sigma$ bands between the $\pi$ band and the lowest C2so band (see Sec. IV A), the broad, generally featureless

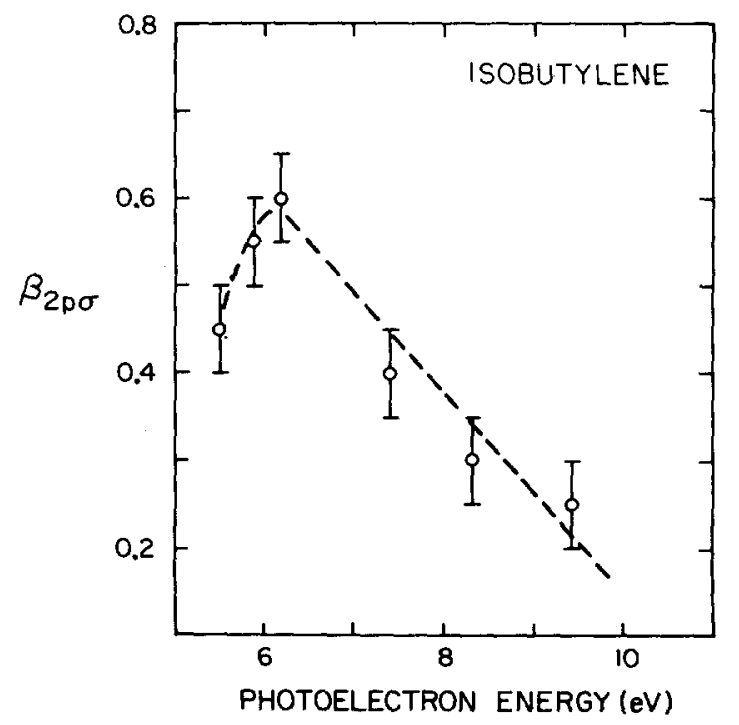

FIG. 6. Variation of the asymmetry parameter, $\beta$, with photoelectron energy, over the $2 p \sigma$ region of the isobutylene photoelectron spectrum. 
TABLE III. Characteristics of the photoelectron spectra of trimethylethylene. ${ }^{a}$

\begin{tabular}{|c|c|c|c|c|}
\hline $\begin{array}{c}\text { Vertical I. P. } \\
(\mathrm{eV})\end{array}$ & $\begin{array}{c}\text { Orbital } \\
\text { symmetry }^{b}\end{array}$ & $\begin{array}{c}\text { Orbital } \\
\text { description }\end{array}$ & $\begin{array}{c}\text { Range of } \beta \\
\text { across band }\end{array}$ & Vertical $\beta^{\mathrm{c}}$ \\
\hline 8.86 & $4 a^{\prime \prime}$ & $\pi$ & $0.67 \pm 0.07-0.80 \pm 0.05$ & $0.80 \pm 0.05$ \\
\hline 11.35 & $16 a^{\prime d}$ & $\mathrm{C} 2 p \sigma$ & $0.20 \pm 0.05-0.30 \pm 0.08$ & $0.25 \pm 0.05$ \\
\hline 12.55 & $15 a^{\prime d}$ & $\mathrm{C} 2 p \sigma$ & $0.27 \pm 0.05-0.43 \pm 0.06$ & $0.30 \pm 0.05$ \\
\hline 12.90 & $a^{\prime}$ & $\mathrm{C} 2 p \sigma$ & $0.36 \pm 0.05-0.47 \pm 0.04$ & $0.40 \pm 0.05$ \\
\hline$(13.6)^{\ominus}$ & $2 a^{\prime \prime}, 3 a^{\prime \prime}, a^{\prime}$ & $\begin{array}{l}\mathrm{C} 2 p \sigma ; \text { in plane } \\
\text { and } \pi\left(\mathrm{CH}_{3}\right)^{f}\end{array}$ & $0.34 \pm 0.06-0.62 \pm 0.07$ & $0.40 \pm 0.05$ \\
\hline 14.73 & $a^{\prime}$ & $\mathrm{C} 2 p \sigma$ & $0.48 \pm 0.08-0.76 \pm 0.07$ & $0.60 \pm 0.10$ \\
\hline 15.60 & $1 a^{\prime \prime}$ & $\mathrm{C} 2 p o ; \pi\left(\mathrm{CH}_{3}\right)^{\mathrm{f}}$ & $0.29 \pm 0.07-0.56 \pm 0.07$ & $0.45 \pm 0.10$ \\
\hline 16.83 & $a^{\prime}$ & $\mathrm{C} 2 \operatorname{so}$ & $0.43 \pm 0.06-0.70 \pm 0.10$ & $0.50 \pm 0.10$ \\
\hline
\end{tabular}

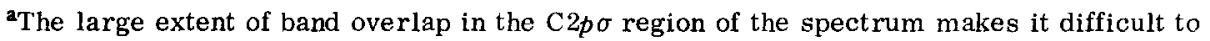
identify all nine features expected in the range 10.6-16.6 eV (see Sec. III C).

${ }^{b}$ The overall symmetry of trimethylethylene is $C_{s}$.

${ }^{c}$ Evaluated at indicated ionization potential.

${ }^{d}$ Reference 28 .

Estimated from slope break.

${ }^{1}$ Assignments of out-of-plane structure based upon LCBO eigenvalues and eigenfunctions (see Sec. IV C).

region between 11.7 and $15.5 \mathrm{eV}$ may contain nine extensively overlapping features.

In Table IV we list selected I.P.'s for tetramethylethylene since, as for trimethylethylene, extensive band overlap is expected in the C2po region of the spectrum, making the identification of all the corresponding bands very difficult. The table also contains orbital characteristics and values of $\beta$. The $\beta$ value for the $\pi$ ionization is now only slightly higher than that measured at the peak of the lowest lying $2 p \sigma$ ionization. A plot of $\beta$ values at selected peaks and shoulders of the $2 p \sigma$ region between 10 to $16 \mathrm{eV}$ vs photoelectron energy is displayed in Fig. 8. Once more, the points lie on a straight line with one exception. The $\beta$ corresponding to the peak at $15.85 \mathrm{eV}$ ionization potential is anomalously low, for reasons given in Sec. IV C.

\section{DISCUSSION}

\section{A. Number of expected C2po bands}

The number of valence C2po bands in the series of molecules considered in this paper can be obtained without any energy eigenvalue calculations as follows. Noncyclic mono-olefins have the generic formula $\mathrm{C}_{n} \mathrm{H}_{2 n}$. There are four valence electrons per carbon and one per hydrogen in these compounds. The total of $6 n$ such electrons is distributed into $3 n$ bonds, resulting in $3 n$ valence photoelectron bands. The C2s $\sigma$ bands in general occur at higher I. P. than the $\pi$ 's or C2po's. ${ }^{10(b),(c)}$ The C2so orbitals form a special class of hydrocarbon molecular orbitals which are principally linear combinations of the C2s atomic orbitals. The number of independent linear combinations of such orbitals is $n$, resulting in $n \mathrm{C} 2 s \sigma$ valence photoelectron bands. ${ }^{19(c)}$ Thus, the number of the remaining $\mathrm{C} 2 p \pi$ and $\mathrm{C} 2 p \sigma$ bands must be $2 n$. For each of the olefins studied here, the I.P. of the single $\pi$ orbital is more than $1 \mathrm{eV}$ below that of the lowest $\sigma \mathrm{I}$. P. In addition, the lowest C2s $\sigma \mathrm{I}$. P. is greater than the highest C2po one. ${ }^{19(b),(c)}$ Under these conditions, the structure in the $\mathrm{C} 2 p \sigma$ region must include $2 n-1$ bands and is bracketed between the $\pi$ and the lowest C2s $\sigma$ bands. Consequently, there should be three $\mathrm{C} 2 p \sigma$ bands in ethylene, seven in isobutylene, nine in trimethylethylene, and 11 in tetramethylethylene. This is the basis for the enumeration of bands in Sec. III.

\section{B. Electron kinetic energy dependence of $\beta$}

Bands arising from ionization of orbital electrons of similar bonding character $(\pi, 2 p \sigma$, or $2 s \sigma)$ might be expected to show similarities in $\beta$. The following information is available for investigation of these similarities: variation of $\beta$ across individual isolated bands, variation of $\beta$ among photoelectron bands of similar type, and a comparison of $\beta$ among corresponding bands of structurally similar molecules. We proceed to analyze this information.

\section{1. $\pi$ bands}

Examination of Figs. 2-5 reveals that $\beta$ varies with electron kinetic energy across the $\pi$ band. In the ab-

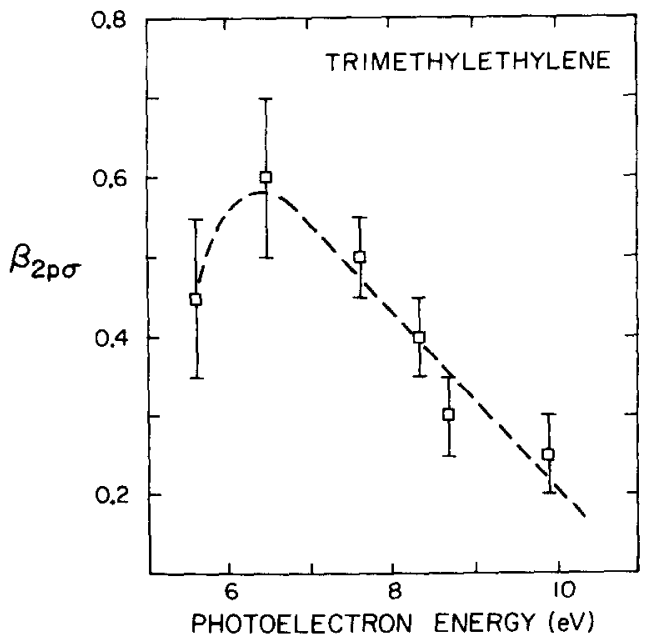

FIG. 7. Variation of the asymmetry parameter, $\beta$, with photoelectron energy, over the $2 p \sigma$ region of the trimethylethylene photoelectron spectrum. 
TABLE IV. Characteristics of the photoelectron spectra of tetramethylethylene. ${ }^{a}$

\begin{tabular}{|c|c|c|c|c|}
\hline $\begin{array}{l}\text { Vertical I. P. } \\
(\mathrm{eV})\end{array}$ & $\begin{array}{c}\text { Orbital } \\
\text { symmetry }^{b}\end{array}$ & $\begin{array}{c}\text { Orbital } \\
\text { description }\end{array}$ & $\begin{array}{l}\text { Range of } \beta \\
\text { across band }\end{array}$ & Vertical $\beta^{\mathrm{e}}$ \\
\hline 8.44 & $2 b_{34}$ & $\pi$ & $0.55 \pm 0.05-0.60 \pm 0.05$ & $0.60 \pm 0.05$ \\
\hline 10.96 & $4 b_{3 e} \mathrm{~d}$ & $\mathrm{C} 2 p \sigma$ & $0.17 \pm 0.04-0.29 \pm 0.05$ & $0.25 \pm 0.05$ \\
\hline 12.71 & $6 a_{g},{ }^{\mathrm{d}} a^{\prime}$ & $\mathrm{C} 2 p o$ & $0.23 \pm 0.07-0.43 \pm 0.06$ & $0.40 \pm 0.05$ \\
\hline$(13.5)^{\circ}$ & $1 b_{1 g}, 1 a_{w}, 1 b_{2 \xi}, a^{\prime}$ & $\mathrm{C} 2 p \sigma ; \pi\left(\mathrm{CH}_{3}\right)$ & $0.42 \pm 0.04-0.50 \pm 0.05$ & $0.50 \pm 0.10$ \\
\hline$(14.2)^{\circ}$ & $a^{\prime}$ & $\mathrm{C} 2 p \sigma$ & $0.45 \pm 0.07-0.72 \pm 0.06$ & $0.60 \pm 0.10$ \\
\hline 14.85 & $a^{\prime}$ & $\mathrm{C} 2 p \sigma$ & $0.66 \pm 0.04-0.76 \pm 0.08$ & $0.70 \pm 0.10$ \\
\hline 15.85 & $1 b_{3 u}$ & $\mathrm{C} 2 p \sigma ; \pi\left(\mathrm{CH}_{3}\right)$ & $0.29 \pm 0.09-0.55 \pm 0.08$ & $0.40 \pm 0.10$ \\
\hline$(16.4)^{\ominus}$ & $a^{\prime}$ & $\mathrm{C} 2 s \sigma$ & $0.31 \pm 0.09-0.63 \pm 0.09$ & $0.50 \pm 0.10$ \\
\hline
\end{tabular}

${ }^{2}$ The large extent of overlap in the C2po region of the spectrum makes it difficult to identify all 11 features expected in the range $10-16 \mathrm{eV}$ (see Sec. III D).

${ }^{b}$ The overall symmetry of tetramethylethylene is $D_{2 h}$. Most of the in-plane $a^{\prime}$ orbitals were assigned using point group $C_{s}$. They are used to designate collectively the set of orbitals $a_{\varepsilon}, b_{1 u}$, $b_{2 u}, b_{3 z}$ of $D_{2 n}$. All orbitals other than $a^{\prime}$ are out of plane and were assigned using point group $D_{2 h}$ and LCBO eigenfunctions and eigenvalues.

${ }^{c}$ Evaluated at indicated ionization potential.

Reference 28.

-Shoulder.

sence of autoionization, this variation can be interpreted as follows. Within the framework of the Born-Oppenheimer approximation and the Franck-Condon principle, the ratio of the intensities of any two vibrational peaks of a given electronic band should equal the ratio of the corresponding Franck-Condon factors. This implies that this ratio should be independent of detector angle, and therefore that $\beta$ should be the same for all vibrational peaks of an electronic band. ${ }^{29}$ However, photoelectrons corresponding to formation of these different peaks have different kinetic energy and can therefore have slightly different values of $\beta$ due to the variation of this parameter with electron kinetic energy..$^{13}$ We have studied such variations with the present apparatus over the $A^{2} \Pi_{4}$ state in $\mathrm{N}_{2}^{+30}$ and the $A^{2} \Pi$ state in $\mathrm{CO} .{ }^{31}$

The variation of $\beta$ across a given electronic band can be used to infer the variation of $\beta$ for a fixed vibrational transition with electron kinetic energy (i.e., with photon energy) if autoionization does not occur. As seen previously in the $73.6 \mathrm{~nm}$ spectra of $\mathrm{N}_{2}$ and $\mathrm{O}_{2}$, autoionization grossly distorts Franck-Condon envelopes..$^{30,32}$ If the lifetime of an autoionizing state exceeds one molecular rotation, preferred directions in space are averaged out and $\beta$ tends to zero, as Carlson has discussed. ${ }^{33}$ Measured $\beta$ values at photoelectron peaks which are enhanced due to autoionization are closer to zero than values measured in the absence of autoionization. The vibrational envelopes of the first $\pi$ band of isobutylene, trimethylethylene, and tetramethylethylene bear strong resemblance to each other and to those of isomeric butenes studied by Kimura et al. ${ }^{19}$ and White et al. ${ }^{16}$ All these molecules show a minor decrease in $\beta$ with increasing vibrational excitation in the ion. The presence of autoionization usually manifests itself in irregular vibrational envelopes and in a rapid and at times nonmonotonic variation of $\beta$ with vibrational peak. The absence of such effects in these $\pi$ bands suggests that they are not affected by autoionization.

The variation of $\beta$ with electron kinetic energy $\left(E_{\theta}\right)$ across the $\pi$ band of ethylene and all of the methyl-substituted ethylenes is given in Fig. 9 and Table V. We see that $d \beta / d E_{e}$ ranges from $-0.1 \mathrm{eV}^{-1}$ for propylene to $0.4 \mathrm{eV}^{-1}$ for cis-2-butene, with more than half of the values lying between 0.1 and $0.2 \mathrm{eV}^{-1}$. The variability of this derivative parameter among the $\pi$ bands of these related molecules precludes its usefulness as a means of identification of $\pi$ bands. The rather large value of 0.4 $\mathrm{eV}^{-1}$ for $d \beta_{\mathrm{r}} / d E$ in $c i s-2$-butene is matched by an equal value for this parameter in benzene. ${ }^{34}$ Another interest ing $\pi$ electron system which permits an evaluation of the variation of $\beta_{r}$ with $E_{e}$ over a rather wide energy range is 1,3-butadiene. This molecule has two $\pi$ bands, one due to ionization of a $b_{\varepsilon}$ electron with a vertical 1.P. of $9.0 \mathrm{eV}$ and the other from an $a_{\mathrm{u}}$ ionization at $11.5 \mathrm{eV}$. The corresponding values of $\beta$ are 0.95 and 0.75 , respectively. ${ }^{16}$ The slope of $0.08 \mathrm{eV}^{-1}$ for the interpolated line matches the value of $d \beta_{\mathrm{r}} / d E$ across the $b_{8}$ band itself. Therefore, this slope is approximately constant over the wide energy range of $2.5 \mathrm{eV}$.

In order to compare the value of $\beta_{r}$ among the different

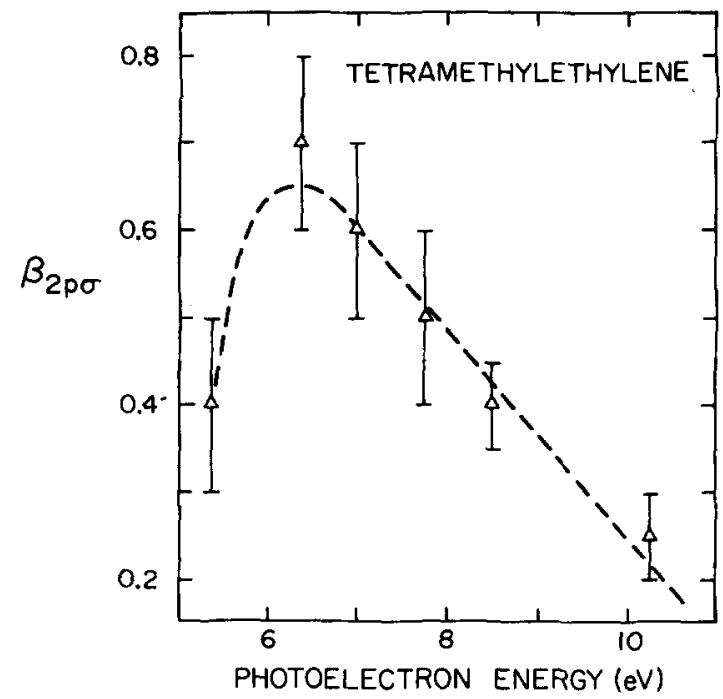

FIG. 8. Variation of the asymmetry parameter, $\beta$, with photoelectron energy, over the $2 p \sigma$ region of the tetramethylethylene photoelectron spectrum. 


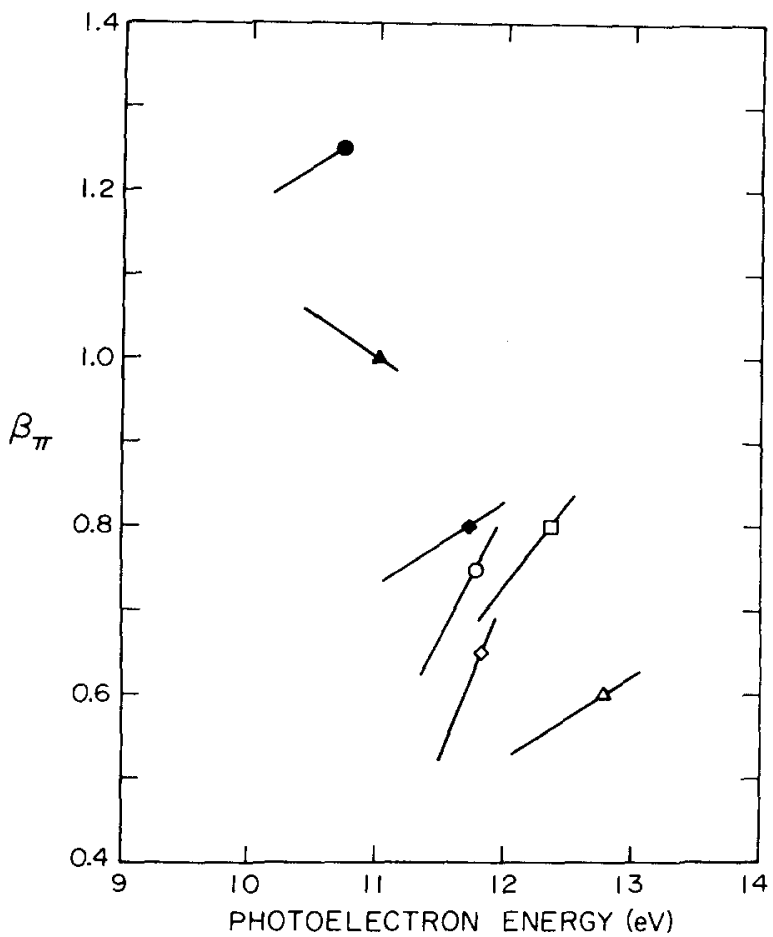

FIG. 9. Variation of the asymmetry parameter, $\beta$, with photoelectron energy, over the $\pi$ band of the photoelectron spectra of the complete set of methyl-substituted ethylenes. Lines represent the variation of $\beta$ over each vibrational envelope. $\bullet$, ethylene. $\Delta$, propylene. $\diamond$, cis-2-butene. $\bullet$, trans-2-butene. $o$, isobutylene. $o, 2$-methyl-2-butene. $\Delta, 2,3$-dimethyl2-butene. Data for propylene, cis-, and trans-2-butene are from Ref. 16 .

related molecules being considered, it would be desirable to do so at the same electron kinetic energy, such as $10.71 \mathrm{eV}(=21.22 \mathrm{eV}$ minus the vertical $\pi$ I.P. of ethylene). In the absence of being able to vary the incident photon energy to achieve this objective, we use instead a linear extrapolation of the $\beta\left(E_{e}\right)$ lines of Fig. 9. The constancy of $d \beta_{r} / d E_{e}$ for 1,3-butadiene over the wide energy range just mentioned indicates that such an extrapolation is reasonable, since the largest extrapolation step for the whole set of molecules being considered is only $1.4 \mathrm{eV}$ (for tetramethylethylene). The corresponding extrapolated values of $\beta$ are given in Table $V$ and displayed in Fig. 10 as a function of the number $n$ of methyl substituents.

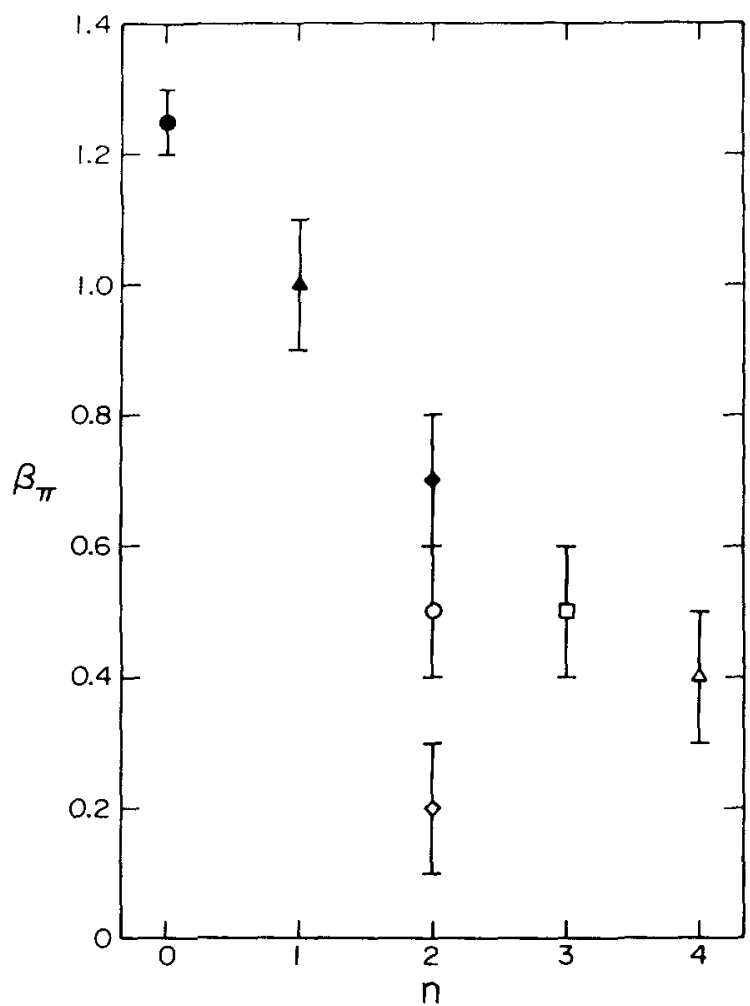

FIG. 10. Variation of the asymmetry parameter, $\beta$, for the $\pi$ band of the photoelectron spectra of the methylated ethylenes, with the number $n$ of methyl substituents. The values of $\beta_{\text {, }}$ were extrapolated to the photoelectron kinetic energy of 10.71 $\mathrm{eV}$ corresponding to the vertical I. P. of ethylene (see Table V).

In comparisons of $\beta_{\pi}$ with or without extrapolation to the same electron kinetic energy we notice that $\beta_{r}$ is a strongly decreasing function of $n$. This was first noted by White $e t a l .{ }^{16}$ in their study of ethylene, propylene, and cis- and trans-2-butene. In contrast to the behavior of $\beta$ for $2 p \sigma$ and $2 s \sigma$ ionizations, values of $\beta$, do not lie within error bars of each other. The extrapolation of $\beta_{\text {r }}$ to $10.71 \mathrm{eV}$ electron energy accentuates the decrease in $\beta_{r}$ with increasing methyl substitution. A possible explanation of the dependence of $\beta$, on $n$ is examined in Sec. IV C.

\section{2. $2 p \sigma$ bands}

For each of the molecules considered, $\beta$ varies slowly with I. P. across the $2 p \sigma$ bands (see Figs. 2-5). There-

TABLE V. Rate of variation of asymmetry parameter with photoelectron energy for the $\pi$ band.

\begin{tabular}{lccccc}
\hline Compound & Orbital & $\begin{array}{l}d \beta / d E_{e} \\
\left(\mathrm{eV}^{-1}\right)\end{array}$ & $\begin{array}{c}\beta \text { extrapolated } \\
\text { to } E_{e}=10.71 \mathrm{eV}\end{array}$ & $f_{a}^{\mathbf{r a}}$ & $\begin{array}{c}\text { LCBO } \\
1-\left|\mathrm{C}_{\boldsymbol{r}}(\mathrm{C}=\mathrm{C})\right|^{2 \mathrm{~b}}\end{array}$ \\
\hline ethylene & $1 b_{3 u}$ & $0.10 \pm 0.05$ & $\mathbf{1 . 2 5} \pm 0.05$ & 0 & 0 \\
propylene & $2 a^{\prime \prime}$ & $-0.10^{\mathrm{c}}$ & $1.0 \pm 0.1$ & 0.2 & 0.14 \\
cis-2-butene & $2 b_{2}$ & $0.4^{\mathrm{c}}$ & $0.2 \pm 0.1$ & 0.9 & 0.20 \\
trans-2-butene & $2 a_{u}$ & $0.1^{\mathrm{c}}$ & $0.7 \pm 0.1$ & 0.5 & 0.20 \\
isobutylene & $2 b_{1}$ & $0.30 \pm 0.05$ & $0.5 \pm 0.1$ & 0.6 & 0.19 \\
trimethylethylene & $4 a^{\prime \prime}$ & $0.20 \pm 0.05$ & $0.5 \pm 0.1$ & 0.6 & 0.23 \\
tetramethylethylene & $2 b_{3 u}$ & $0.10 \pm 0.05$ & $0.4 \pm 0.1$ & 0.7 & 0.26 \\
\hline \hline
\end{tabular}

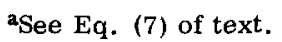

cMeasured from Figs. 2-4 of Ref. 16.

${ }^{b}$ See Sec. IV C 3 and Appendix. 


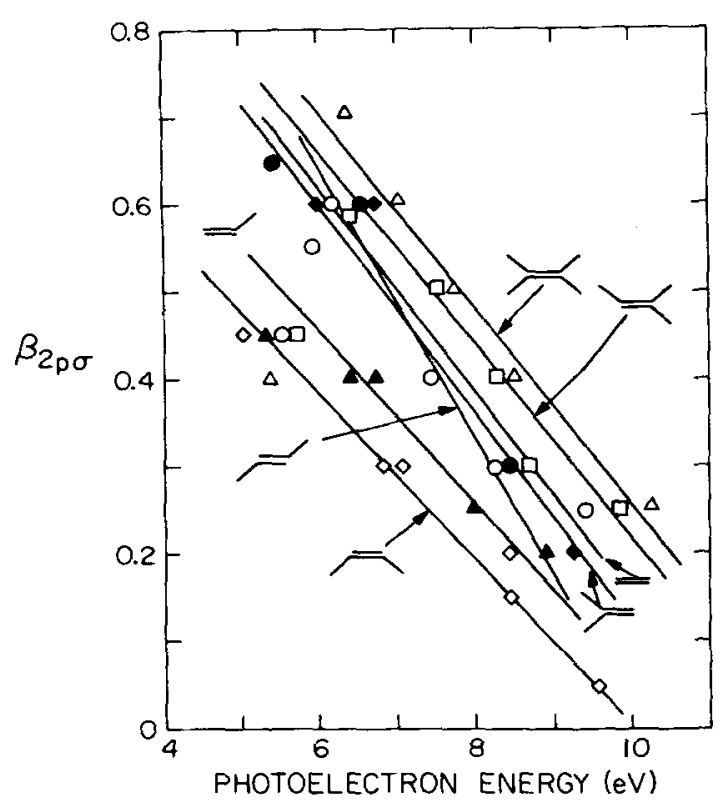

FIG. 11. Variation of the asymmetry parameter, $\beta$, with photoelectron energy, over the $2 p \sigma$ region of the photoelectron spectra of the complete set of methyl substituted ethylenes. Symbols are the same as those of Fig. 9. Data for propylene, cis- and trans-2-butene are from Ref, 16.

fore, it may be assumed that these bands are the result of direct photoionization processes.

As pointed out in Secs. III B-III D and displayed in Figs, $6-8, \beta$ decreases with increasing $E_{e}$ (i. e. , decreasing I. P.) over most of the $2 p \sigma \mathrm{I}$. P. range for isobutylene, trimethylethylene, and tetramethylethylene. White et al. ${ }^{16}$ had previously noticed this trend in ethylene, propylene, and several of the isomeric butenes. Let us attempt to rationalize this rather general behavior of this family of molecules.

Taking isobutylene as a typical case, we expect an assortment of $A_{1}, A_{2}$, and $B_{2}$ excited ionic states to result from the photoionization process. In the orbital approximation, ${ }^{1}$ each band corresponds to the photoejection of an electron from a $\sigma$ molecular orbital. Although belonging to different symmetry groups, these $\sigma$ orbitals all have some $\mathrm{C}-\mathrm{C}$ and some $\mathrm{C}-\mathrm{H}$ bonding character. In the photoionization of atoms, the angular momentum quantum number $l$ of the initial atomic orbital from which the electron is photoejected significantly influences the electron kinetic energy dependence of the corresponding $\beta_{.}^{21}$ For a given principal quantum number, the value of $l$ also determines the number of nodal surfaces of that atomic orbital. Similarly, we expect that for $n l \lambda$ molecular orbitals (such as $2 p \sigma$ ), the point symmetry to which these orbitals belong (and which defines its nodal characteristics) would also strongly influence the dependence of the corresponding $\beta$ on $E_{e}$. We therefore expect that all ionizations from $n l \lambda$ orbitals of a given $\Gamma$ symmetry to have a similar electron energy dependence of $\beta$. For example, $\beta$ for the several $a_{1} 2 p \sigma$ ionizations of isobutylene taken as a group are expected to vary with $E_{\theta}$ in an analogous manner, as would the $2 p \sigma b_{2}$ ionizations. However, there is no reason to expect, on the basis of these arguments, that these $a_{1}$ and $b_{2}$ ionizations should have $\beta\left(E_{e}\right)$ curves similar to one another. Yet, the $\beta$ values for the $a_{1}, a_{2}$, and $b_{2} 2 p \sigma$ ionizations of isobutylene (see Table II) all lie on the same straight line of Fig. 6. A similar behavior is also found in tri- and tetramethylethylene, as indicated in Figs. 7 and 8, respectively.

A related behavior has been observed previously by Kinsinger and Taylor ${ }^{34}$ who measured the photoelectron angular distributions for benzene at the 74.0 and 58.4 $\mathrm{nm}$. They found that for orbitals of the same type (according to the $\pi, r, s, t$ classification of Jonsson and Lindholm ${ }^{35}$ ), the corresponding $\beta$ values can be connected by a smooth curve. Significantly, orbitals within each group were of different group-theoretical designations $\Gamma$. As an example, the $\pi$ orbitals included those of $e_{2 \varepsilon}$ and $a_{2 u}$ symmetry. This is in agreement with our observation that, within a given orbital type ( $2 p \sigma$ for the methylethylenes and $\pi, r, s$, or $t$ for benzene), the electron energy variation of $\beta$ is independent of the symmetry group to which the initial electron molecular orbital belongs.

In order to analyze the behavior of the $\beta_{2 \rho \sigma}\left(E_{e}\right)$ curves for the family of molecules being considered, all of these curves are plotted together in Fig. 11. (For simplicity, we omit the points corresponding to the lowest $E_{e}$ for each of these molecules. These are discussed separately in Sec. IV C.) Results from the study of propylene and cis - and trans-2-butene of White et al. ${ }^{16}$ are included for completeness. Significantly, all lines are parallel and close to one another: The values of $\beta$ for any given $E_{e}$ lie within \pm 0.15 of their average, being almost equal within the experimental error of about 0.1 . This result is striking in view of the diverse collection of molecular symmetry groups $\left(C_{2 v}, C_{2 h}, C_{s}, D_{2 h}\right)$ represented and suggests that the variation of $\beta_{2 p o}$ with $E_{e}$ is very much independent of molecular symmetry. The reason for this behavior may be the planar character of all of the $\sigma$ molecular orbitals from which the electrons are photoejected.

\section{3. $2 s \sigma$ bands}

The similarities observed in the electron energy dependence of $\beta_{x}$ and $\beta_{p \sigma}$ suggest that curves analogous to those of Figs. 9 and 11 for ionization of electrons from carbon $2 s \sigma$ orbitals should show some clear common trends as well.

The assignment of the highest I.P. band in the 58.4 $\mathrm{nm}$ photoelectron spectra as due to ionization of the least tightly bound carbon $2 s$ electron is based on similarities in peak positions for families of hydrocarbons. ${ }^{25,36,37}$ As seen in Figs. 3-5, this band shifts to lower I. P. with increasing molecular size. Because of their higher I. P.'s, more tightly bound carbon $2 s$ bands usually do not appear in $58.4 \mathrm{~nm}$ spectra. ${ }^{25}$ As a result, the variation of $\beta$ with electron energy for carbon $2 s$ bands can only be obtained over the limited energy range provided by the width of a single band. For the series of molecules we studied, poor signal-to-background ratios yield large error bars for $\beta$ and we notice only the absence of gross variations across each $2 s$ band. 


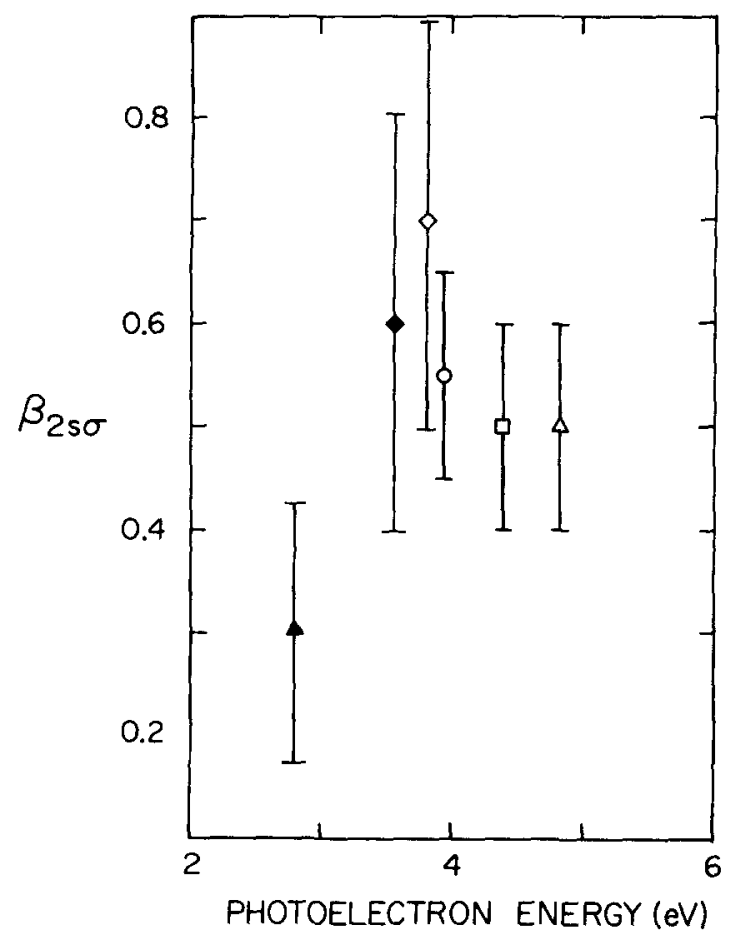

FIG, 12. Variation of the asymmetry parameter, $\beta$, measured at the vertical I. P. of the $2 s \sigma$ band, with photoelectron energy. Symbols have the same meaning as in Fig. 9. No value for ethylene is available. The points for propylene, cisand trans-2-butene are from Ref. 16. The vertical lines are estimates of the measurement uncertainties.

In Fig. 12 we plot the $\beta_{2 s \sigma}$ values as a function of electron energy for the family of six methylated ethylenes, including the three of the present study and the three of White et al. ${ }^{16}$ The error bars for the latter were estimated from their Figs. 2-4. With the exception of propylene, the variation of $\beta_{2 s 0}$ across this series of molecules is apparently slight.

The behavior of $\beta_{2 s 0}$ described above is consistent with the results for the energy dependence of $s$-type bands in the benzene study mentioned earlier. ${ }^{34}$ Reported values of $\beta$ for that molecule of 0.5 for the $2 b_{14}$ band at $15.5 \mathrm{eV}$ I. P. and 0.70 for the $2 e_{2,}$ band at $18.9 \mathrm{eV} \mathrm{I.P.} \mathrm{indicate}$ a slight energy dependence of the asymmetry parameter.

\section{Effects of $\sigma-\pi$ mixing}

We attribute the decrease of $\beta_{\mathrm{r}}$ (see Figs. 9 and 10) with increasing methyl substitution to the mixing of $\pi$ and $\sigma$ orbitals called hyperconjugation ${ }^{38}$ or through-bond interaction. ${ }^{39}$ We believe that this mixing is also responsible for the increase of $\beta_{2 p o}$ with photoelectron energy in the lower energy range (around $5.5 \mathrm{eV}$ ) of Figs. $6-8$.

\section{A quantitative mixing model}

Theories which have been used to study hyperconjugation include the linear combination of bonding orbitals method (LCBO), ${ }^{37,40}$ the structure representation theory, ${ }^{41}$ and the group orbital method ${ }^{27}$ and are all based on the equivalent orbital theory of Hall and Lennard-Jones. ${ }^{26}$ This phenomenological theory has been extensively used previously in photoelectron spectroscopy to predict vertical ionization potentials on the basis of parameters derived from photoelectron spectra of model compounds. ${ }^{37,40,41}$ The orbitals whose electrons are photoejected, corresponding to each band in the spectrum, are interpreted as linear combinations or mixtures of appropriate functions representing structural units in the molecule which transform the same way under one of the several symmetry operations of the molecular symmetry group. For planar unsaturated molecules, these functions, termed "out-of-plane localized orbitals" by Kimura et al.,$^{19}$ represent $\pi$ bonds and linear combinations of $\mathrm{CH}$ bonds. The latter, called $\pi\left(\mathrm{CH}_{3}\right)$, are antisymmetric under reflection in the molecular plane and take the form ${ }^{40}$

$\pi\left(\mathrm{CH}_{3}\right)=\frac{1}{\sqrt{2}}\left(\mathrm{CH}^{1}-\mathrm{CH}^{2}\right) \cos \gamma+\frac{1}{\sqrt{6}}\left(\mathrm{CH}^{1}+\mathrm{CH}^{2}-2 \cdot \mathrm{CH}^{3}\right) \sin \gamma$,

where $\gamma$ is a phase angle related to the rotational orientation of the $\mathrm{CH}_{3}$ structure and the $\mathrm{CH}^{i}(i=1,2,3)$ are functions which represent the three $\mathrm{CH}$ bonds of a methyl group. The theory yields the secular determinantal equation

$$
\left|E_{i j}-\mu \delta_{i j}\right|=0 \text {, }
$$

where $E_{i}$ are energy parameters associated with structural units in the molecule and are obtained from spectral peak positions of model compounds, $\mu$ represents an eigenvalue, and $\delta_{i j}$ is the usual Kronecker delta symbol. As an example, the $E$ matrix and the functions representing structural units for tetramethylethylene are described in the Appendix.

The relevant parameters for the methyl-substituted ethylenes are the energies of the carbon-carbon double bonds $(d)$, of the $\pi\left(\mathrm{CH}_{3}\right)$ structural units $(m)$, of the interaction of a $\pi\left(\mathrm{CH}_{3}\right)$ unit with a carbon-carbon double bond $(x)$, and of the interaction of two $\pi\left(\mathrm{CH}_{3}\right)$ structures attached to the same $s p^{2}$ hybridized carbon $(y)$. Uncertainties in the band-weighted I.P. of methane have yielded a wide variation of the parameter $m$ (13.7-14.3 $\mathrm{eV}) .^{19,27,41}$ Typically, ${ }^{40-42}$ the other parameters have the values

$$
\begin{aligned}
& d=10.51 \mathrm{eV} \\
& x=1.5-1.75 \mathrm{eV} \\
& y \sim 0.2 \mathrm{eV} .
\end{aligned}
$$

A list of sample eigenvalues and eigenfunctions we obtained by this approach is given in Table VI.

We find trends in the eigenvalues and eigenfunctions which are relatively insensitive to the choice of parameters and are expected to be valid even if the collection of parameters used is not completely optimized for fits to peak positions in the photoelectron spectra of related compounds. Conclusions which we derive from these calculations are: (1) The $\pi$ ionization potential, corresponding to the lowest eigenvalue, is lowered with increasing methyl substitution. (2) The largest eigenvalue for out-of-plane structural units is typically in the range $14.5-16.5 \mathrm{eV}$, depending on the choice of parameters. It increases with increasing methyl substitution. 
TABLE VI. Eigenvalues and eigenvectors of LCBO model.

\begin{tabular}{|c|c|c|c|c|c|c|c|}
\hline \multirow[b]{2}{*}{ Molecule } & \multirow[b]{2}{*}{ Orbital } & \multirow{2}{*}{$\begin{array}{c}\text { Eigenvalue }^{a} \\
(\mathrm{eV})\end{array}$} & \multicolumn{5}{|c|}{ Subunit coefficient in eigenvector ${ }^{\mathrm{a}, \mathrm{b}}$} \\
\hline & & & $\mathrm{C}_{\boldsymbol{r}}(\mathrm{C}=\mathrm{C})$ & $\mathrm{C}_{\pi}\left(\mathrm{CH}_{3}^{(1)}\right)$ & $\mathrm{C}_{\mathbf{r}}\left(\mathrm{CH}_{3}^{(2)}\right)$ & $\mathrm{C}_{8}\left(\mathrm{CH}_{3}^{(3)}\right)$ & $\mathrm{C}_{\pi}\left(\mathrm{CH}_{3}^{(4)}\right)$ \\
\hline \multirow[t]{2}{*}{ propylene } & $2 a^{\prime \prime}$ & 9.812 & 0.9289 & -0.3704 & & & \\
\hline & $1 a^{\prime \prime}$ & 14.898 & 0.3704 & 0.9289 & & & \\
\hline \multirow[t]{3}{*}{ cis-2-butene } & $2 b_{2}$ & 9.268 & 0.8938 & -0.3171 & & & -0.3171 \\
\hline & $1 a_{2}$ & 14.200 & 0.0 & 0.7071 & & & -0.7071 \\
\hline & $1 b_{2}$ & 15.442 & 0.4485 & 0.6319 & & & 0.6319 \\
\hline \multirow[t]{3}{*}{ trans-2-butene } & $2 a_{u}$ & 9.268 & 0.8938 & -0.3171 & & -0.3171 & \\
\hline & $1 b_{\xi}$ & 14.200 & 0.0 & 0.7071 & & -0.7071 & \\
\hline & $1 a_{u}$ & 15.442 & 0.4485 & 0.6319 & & 0.6319 & \\
\hline \multirow[t]{3}{*}{ isobutylene } & $2 b_{1}$ & 9.307 & 0.8994 & -0.3091 & -0.3091 & & \\
\hline & $1 a_{2}$ & 14.000 & 0.0 & 0.7071 & -0.7071 & & \\
\hline & $1 b_{1}$ & 15.603 & 0.4372 & 0.6360 & 0.6360 & & \\
\hline \multirow[t]{4}{*}{ trimethylethylene } & $4 a^{\prime \prime}$ & 8.838 & 0.8755 & -0.2754 & -0.2754 & -0.2858 & \\
\hline & $3 a^{\prime \prime}$ & 14.000 & 0.0 & 0.7071 & -0.7071 & 0.0 & \\
\hline & $2 a^{\prime \prime}$ & 14.263 & 0.0302 & -0.3862 & -0.3862 & 0.8371 & \\
\hline & $1 a^{\prime \prime}$ & 16.009 & 0.4822 & 0.5244 & 0.5244 & 0.4664 & \\
\hline \multirow[t]{5}{*}{ tetramethylethylene } & $2 b_{3 u}$ & 8.451 & 0.8619 & -0.2536 & -0.2536 & -0.2536 & -0.2536 \\
\hline & $1 b_{18}$ & 14.000 & 0.0 & 0.5000 & -0.5000 & -0.5000 & 0.5000 \\
\hline & $1 a_{u}$ & 14.00 & 0.0 & 0.5000 & -0.5000 & 0.5000 & -0.5000 \\
\hline & $1 b_{28}$ & 14.400 & 0.0 & 0.5000 & 0.5000 & -0.5000 & -0.5000 \\
\hline & $1 b_{34}$ & 16.459 & 0.5071 & 0.4310 & 0.4310 & 0.4310 & 0.4310 \\
\hline \multicolumn{3}{|c|}{$\begin{array}{l}\text { 2Using the parameters: } \begin{aligned} d & =10.51 \mathrm{eV} \\
m & =14.2 \mathrm{eV} \\
x & =1.75 \mathrm{eV} \\
y & =0.2 \mathrm{eV}\end{aligned}\end{array}$} & \multicolumn{2}{|c|}{ and the geometry } & & & \\
\hline
\end{tabular}

When two or more methyl groups are substituted on the ethylene frame, all other eigenvalues cluster about $m$. (4) All but the lowest eigenvalue correspond to electronic bands within the carbon $2 p \sigma$ region of the photoelectron spectrum. (5) The eigenfunctions corresponding to the highest and lowest eigenvalues have nonzero coefficients for $\pi(\mathrm{C}=\mathrm{C})$ and for the various $\pi\left(\mathrm{CH}_{3}\right)$. All other eigenfunctions have either zero coefficient of $\pi(C=C$ ) (as a consequence of the molecular symmetry) or a small value for this coefficient (in the case of the $2 a^{\prime \prime}$ orbital of trimethylethylene). (6) With increasing methyl substitution, the coefficient of $\pi\left(\mathrm{CH}_{3}\right)$ increases for the eigenfunction corresponding to the lowest eigenvalue (largely $\pi$ ionization) with increasing methyl substitution. (7) Conversely, with increasing methyl substitution, the coefficient of $\pi(C=C)$ increases for the eigenfunction corresponding to the highest eigenvalue.

Eigenvalue trends have been tested against experimental vertical ionization potentials of the $2 p \pi$ and $2 p \sigma$ bands in the photoelectron spectra of methylated ethylenes. ${ }^{18,27}$ Much of the experimental $\sigma$ structure in the photoelectron spectra overlaps grossly, and it is very difficult to unambiguously assign particular features of the latter to loss of out-of-plane orbital electrons. In isobutylene, trimethylethylene, and tetramethylethylene, the highest eigenvalue is predicted to lie at the higher I. P. end of the $2 p \sigma$ region of the spectrum where overlap with other $2 p \sigma$ bands is minimized.

\section{Out-of-plane ionizations lacking $\pi(\mathrm{C}=\mathrm{C})$ contributions}

In the previous subsection we have shown that the mixing model predicts that in the central part of the $2 p o$ region of the photoelectron spectrum (around 14.2 eV I. P. or $7 \mathrm{eV}$ photoelectron energy), the out-of-plane ionizations do not contain $\pi(\mathrm{C}=\mathrm{C})$ contributions. This facilitates the analysis of this spectral region. In it, $\beta_{2 p o}$ varies slowly and monotonically with photoelectron energy (see Figs. 6-8) for the complete set of methylated ethylenes. The mixing model predicts the existence of some out-of-plane orbital electron ionizations in this region. Their number, however, is smaller than the total number of bands predicted (see Sec. IV A), which means that several in-plane orbital ionization bands also occur in the same region of the spectrum. For example, for isobutylene, we have two out-of -plane bands in the ionization energy region between the lowest $\pi$ ionization and the lowest $2 s \sigma$ ionization, whereas a total of seven bands is predicted. Therefore, there exists an additional five in-plane bands, some of which must overlap significantly with the out-of-plane one around $14.2 \mathrm{eV}$. As a result, one might expect a rather sharp change of $\beta$ with photoelectron energy across a region of the photoelectron spectrum over which the relative contributions of the outof -plane and in-plane bands change significantly. That such a sharp change is not observed in the $I$. P. region around $14.2 \mathrm{eV}$ is suggestive that these two kinds of bands have $\beta$ values which are very close to one another. 
TABLE VII. Variation of asymmetry parameter, $\beta$, over $2 p \sigma$ region.

\begin{tabular}{lccccc}
\hline Compound & $\begin{array}{c}\text { Pseudo- } \pi \\
\text { orbital }\end{array}$ & $\begin{array}{c}\text { I. P., }{ }^{2} \text { of } \\
\text { corresponding } \\
\text { band }\end{array}$ & $d \beta / d E_{e}$ & $\beta_{\text {extrap }}-\beta_{\text {expt }}$ & $\left|\mathrm{C}_{\pi}(\mathrm{C}=\mathrm{C})\right|^{2}$ \\
\hline isobutylene & $1 b_{1}$ & $15.7^{\mathrm{C}}$ & -0.12 & $0.20 \pm 0.05$ & 0.19 \\
trimethylethylene & $1 a^{\prime \prime}$ & 15.60 & -0.11 & $0.25 \pm 0.10$ & 0.23 \\
tetramethylethylene & $1 b_{3 \text { s }}$ & 15.85 & -0.12 & $0.40 \pm 0.10$ & 0.26 \\
\hline
\end{tabular}

avertical, in $\mathrm{eV}$.

${ }^{\mathrm{c}}$ Measured at a shoulder.

beasured from variation over other $2 p \sigma$ structure.

\section{Out-of-plane ionizations having $\pi(\mathrm{C}=\mathrm{C})$ contributions}

We now consider the lowest I. P. $2 p \pi$ bands and the high I. P. region of the $2 p \sigma$ bands. The discussion of Sec. IV C1 showed that, according to the mixing model, these bands involve ionization from orbitals which contain both $\pi(\mathrm{C}=\mathrm{C})$ and $\pi\left(\mathrm{CH}_{3}\right)$ contributions (see Table V). Let us analyze the effect of this mixing on $\beta$ values.

The resonant mixing of the $\pi\left(\mathrm{CH}_{3}\right)$ and the $\pi(\mathrm{C}=\mathrm{C})$ elec trons can be crudely described by writing the resulting hybrid orbital $\psi$ as a linear combination of the orbitals $\phi_{1}$ and $\phi_{2}$ which correspond respectively to those types of electrons,

$$
\psi=\mathrm{C}_{1} \phi_{1}+\mathrm{C}_{2} \phi_{2}
$$

Within the single configuration linear combination of atomic orbitals - molecular orbital (LCAO-MO) approximation, the angular dependence of the photoelectron differential cross section comes from the square of the absolute value of the matrix element $\left\langle\psi_{\boldsymbol{g}}|\hat{\boldsymbol{\epsilon}} \cdot \mathbf{r}| \mathbf{C}_{1} \phi_{1}\right.$ $\left.+C_{2} \phi_{2}\right\rangle$, where $\psi_{e}$ is the wave function of the ejected photoelectron, $\hat{\boldsymbol{\epsilon}}$ is the unit vector in the polarization direction of the light beam, and $\boldsymbol{r}$ is the position vector of the electron. ${ }^{21}$ Multiplying this transition amplitude by its complex conjugate leads to four terms, two of which are interference cross terms which we ignore in this simplified analysis. Such an approximation is valid at high energies. ${ }^{43}$ Of the other two terms, one contains the angular dependence parameter $\beta_{1}$ of the photoelectrons ejected from the $\phi_{1}$ orbital and the other contains $\beta_{2}$ which is associated with photoionization from the $\phi_{2}$ orbital. The resulting value of $\beta$ from this hybrid orbital $\psi$ will, as a result, be a linear combination of these two $\beta$ 's,

$$
\beta=\frac{\left|C_{1}\right|^{2} \sigma_{1} \beta_{1}+\left|C_{2}\right|^{2} \sigma_{2} \beta_{2}}{\left|C_{1}\right|^{2} \sigma_{1}+\left|C_{2}\right|^{2} \sigma_{2}},
$$

where $\sigma_{1}$ and $\sigma_{2}$ are the corresponding integral photoionization cross sections corresponding to the orbitals $\phi_{1}$ and $\phi_{2}$. We take for $\beta_{2}$ the value for the vertical $\mathrm{C}-\mathrm{C} \pi$ band in ethylene which is 1.25 at $E_{\theta}=10.71 \mathrm{eV}$. The value of $\beta_{1}$ for the $\pi\left(\mathrm{CH}_{3}\right)$ orbital is obtained from extrapolating the $\beta$ values for the $2 p \sigma$ region of the molecule being considered to the photoelectron energy of the mixed orbital in question. Under conditions for which the cross terms $C_{q} C_{\sigma}$ can be neglected, the expression for $\beta$ at photoelectron energy $E_{\theta}$ for a molecule obtained from ethylene by replacing $n \mathrm{H}$ atoms by methyls becomes

$$
\beta\left(n, E_{\theta}\right)=f_{r}(n) \beta_{r}\left(0, E_{\theta}\right)+f_{\sigma}(n) \beta_{\sigma}\left(0, E_{\theta}\right) .
$$

Here, $f_{s}$ and $f_{\sigma}$ are fractions associated with the amount of $\pi$ and $\sigma$ character, respectively, of the molecular orbital in question, and add up to unity. $\beta_{\mathrm{r}}\left(0, E_{g}\right)$ and $\beta_{\sigma}\left(0, E_{g}\right)$ are, respectively, the values of $\beta$ for ionization of the $\pi$ and $\sigma$ bands of ethylene [for which there is no mixing between $\pi(\mathrm{C}=\mathrm{C})$ and $\pi\left(\mathrm{CH}_{3}\right)$ structures since the latter are absent]. According to the LCBO approximation considered above, the value of $f_{\mathrm{r}}(n)$ is equal to the $\left|\mathrm{C}_{\pi}(\mathrm{C}=\mathrm{C})\right|^{2}$ of Table VI, and is therefore independent of photoelectron energy.

For ionization of the lowest $\pi$ orbital of the methylated ethylenes, Eq. (6) furnishes

$$
f_{\sigma}^{\pi}(n)=\frac{\beta^{\mathrm{r}}\left(n, E_{\theta}\right)-\beta_{\mathrm{r}}\left(0, E_{\theta}\right)}{\beta_{\mathrm{\sigma}}\left(0, E_{e}\right)-\beta_{\mathrm{s}}\left(0, E_{e}\right)},
$$

where the superscript $\pi$ refers to ionization of the $\pi$ electron of the $n$-methylated ethylene. We would like to compare the values of $f_{\sigma}^{\pi}(n)$ obtained from our measurements of $\beta^{\prime}(n, E)$ extrapolated to $E=10.71 \mathrm{eV}$ and Eq. (7) with the LCBO values. Since we know that $\beta_{\mathrm{r}}(0$, $10.71 \mathrm{eV})=1.25$ (see Table V), we need only to estimate $\beta_{o}(0,10.71 \mathrm{eV})$. Such an estimation can be obtained by extrapolating the mean of the band of lines of Fig. 11 to $E=10.71 \mathrm{eV}$. This furnishes a value of about 0.06 . This and Eq. (7) yield the values of $f_{\mathrm{a}}^{*}$ given in the fifth column of Table $\mathrm{V}$. In the sixth column, the value of $1-\left|C_{r}(C=C)\right|^{2}$ obtained from the LCBO calculations summarized in Table VI are listed. With the exception of cis-2-butene, the values in both these columns increase monotonically with degree of methylation. Therefore, although this model is very crude and quantitatively inaccurate, it does explain Fig. 10 qualitatively by attributing the decrease of $\beta^{2}(n, 10.71 \mathrm{eV})$ with $n$ to increased $\sigma-\pi$ mixing.

We now attempt to use this mixing model to explain the increase of $\beta_{2 p \sigma}$ with photoelectron energy around $E_{\mathrm{s}}=5.5 \mathrm{eV}$ displayed in Figs. 6-8. For ionization of these $\sigma$ bands, Eq. (6) yields

$$
f_{s}^{\sigma}(n)=\frac{\beta_{\sigma}\left(0, E_{\theta}\right)-\beta^{\sigma}\left(n, E_{\theta}\right)}{\beta_{\sigma}\left(0, E_{\theta}\right)-\beta_{r}\left(0, E_{\theta}\right)} \text {. }
$$

We obtain a crude estimate of $\beta_{\sigma}(0,5.5 \mathrm{eV})$ by linea rly extrapolating the curves of Fig. 9 for $E_{8}>7 \mathrm{eV}$ down to $E_{\mathrm{a}}=5.5 \mathrm{eV}$. The differences between those extrapolated values and the measured values of $\beta_{2 p o}$ at that energy are given in Table VII, together with other relevant information about the $2 p \sigma$ bands for the molecules we studied. In order to go through an argument similar to that for ionization of the $\pi$ band given above, we also need to 
estimate $\beta_{r}(0,5.5 \mathrm{eV})$. There is no way of making such an estimation from presently available data, and as a result we resort to a simpler qualitative argument. From the fifth column of Table VII it can be seen that the numerator of the right hand side of Eq. (8) increases with $n$. The last column of that table also shows that $f_{\mathrm{r}}^{0}(n)=\left|\mathrm{C}_{\mathbf{g}}(\mathrm{C}=\mathrm{C})\right|^{2}$ also increases with that parameter. The simultaneous validity of these two properties, together with $\mathrm{Eq}$. (8), requires that the denominator of the right hand side of that equation be positive. Therefore, if for ethylene we have $\beta_{r}<\beta_{\sigma}$ at $E_{g}=5.5 \mathrm{eV}$, the $\sigma-\pi$ mixing model explains the increase of $\beta_{2 p_{0}}$ with $E_{e}$ in this energy region. A test of this model would be to measure $\beta_{\text {r }}$ for ethylene using a NeI resonance lamp $(h \nu=16.8 \mathrm{eV})$ which corresponds to $E_{a}=6.3 \mathrm{eV}$. According to Figs. 6-8 and the mixing model we should get not only $\beta_{\mathrm{r}}<\beta_{2 p \sigma}$ but also $\beta_{r}<0.4$.

Independently of this mixing model, we can estimate the value of $\beta_{s}$ for ethylene near $5.5 \mathrm{eV}$ photoelectron energy on the basis of previous calculations and experiments for related carbon-containing molecules. Kinsinger and Taylor ${ }^{34}$ measured a $\beta_{\text {r value of }}-0.5$ for the benzene $\pi$ orbitals in this energy region. Lohr's calculation $^{44}$ using $C_{2}$ as a model for ethylene gives a $\beta_{r}$ value near -0.8 . This value should be shifted upwards on the basis of his error of $0.5-0.8$ at an electron energy of $10.7 \mathrm{eV}$. Kennedy and Manson's $\beta$ for the carbon atomic $2 p$ subshell $^{45}$ goes to zero at threshold and rises with increasing electron energy at a rate of about $0.1 \mathrm{eV}^{-1}$.

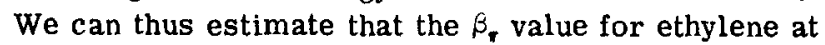
about $E=5.5 \mathrm{eV}$ should be close to zero. This value is lower than that of $\beta_{\sigma}(0,5.5 \mathrm{eV})$ and is consistent with the mixing model.

\section{v. CONCLUSIONS}

From first principles, we would expect the necessity to measure photoelectron angular distributions at a number of photon wavelengths in order to determine the electron energy variation of $\beta$. For polyatomic molecules, the photoelectron angular distributions measured at a single photon energy incorporate much of this information already. If we are judicious, we can infer the variation of $\beta$ across vibrational envelopes. This is so because, in the absence of autoionization, the variation of $\beta$ across a vibrational envelope is almost equivalent to its photoelectron energy dependence. This seems to be the case, in the present studies, for the $\pi$-band ionizations of ethylene and its methyl-substituted derivatives. Also, the variation of $\beta$ within a set of ionizations of closely related orbitals should reflect the energy variations for any of the members of the set. For example, the several $2 p \sigma$ bands of each of the methyl substituted ethylenes were found to behave, in their photoelectron energy dependence of $\beta$, as if they constituted a single band, in spite of the fact that they do not necessarily have the same group theoretical symmetry designation.

The values of $\beta$ are sensitive to the presence of through-bond mixing of orbitals of different types. Measured values of $\beta$ in these mixed systems take on values intermediate between $\beta$ values measured for unmixed systems. Therefore, the deviation from the unmixed value of $\beta$ for the principal subunit, as the deviations of $\beta_{r}$ for the set of methyl-substituted ethylenes from the measured ethylene value, can be used to infer the extent of through-bond $\sigma-\pi$ mixing. The effects of mixing are reciprocal. The $\beta$ values for the $\sigma$ region are perturbed by the $\pi$ orbitals as well.

In order that these preliminary conclusions, based as they are on photoelectron spectra and $\beta$ values obtained at a single photon energy, be adequately tested, it would be highly desirable to perform experiments using a variable photon energy source, analogous to the synchrotron radiation studies of oxygen done by McCoy et al. ${ }^{46}$ The apparatus used in such experiments should have a very high collection efficiency, since under equivalent experimental conditions the counting rates for hydrocarbons of the type considered in the present paper are much smaller than those for diatomic molecules.

In addition, theoretical calculations of reliable quality of the dependence of $\beta$ on photon energy and on orbital type would obviously be very useful. Related theoretical studies on simple systems have recently been performed. ${ }^{47,48}$ However, for the kinds of molecules studied in the present paper, the calculations needed may not be feasible for some time yet. Experimental measurements, including the present ones, may help guide such calculations.

\section{APPENDIX}

We illustrate in this appendix the calculation of the eigenvalues $\mu$ for the out-of-plane orbitals of tetramethylethylene. Using the methyl labeling of the methylsubstituted ethylenes given at the bottom of Table VI, we designate the functions which represent the "out-of plane localized orbitals" as $\pi\left(\mathrm{CH}_{3}^{(i)}\right), i=1,2,3,4$. Those characteristic groups interact with the ethylenic $\pi$ orbital labeled $\pi(C=C)$.

The energy matrix $E_{i}$ is symmetric and has diagonal elements which correspond to the I. P.'s of the three functional groups. Its elements are obtained from an equivalent orbital ${ }^{28}$ analysis of appropriately chosen related hydrocarbons: ethylene, methane, isobutylene, 1,3-butadiene, and 1,4-cyclohexadiene. $E_{11}(=d)$ is the I. $P$. of the $\pi(C=C)$ group and is set equal to the $\pi$ band vertical 1.P. of ethylene. $E_{i i}(=m), i=2,3,4,5$ is the I. P. of the $\pi\left(\mathrm{CH}_{3}\right)$ group and is set equal to a bandweighted average of the $t_{2}$ electron ionization band ${ }^{19,27,41}$ of methane. The off-diagonal elements $E_{i}$, represent the interaction energies between the five characteristic groups. $E_{1 i}(=x), i=2,3,4,5$ is the interaction energy between the $\pi\left(\mathrm{CH}_{3}^{(6)}\right)$ and the $\pi(\mathrm{C}=\mathrm{C})$ orbitals. It is set equal to twice the difference between the average of the lowest two I.P.'s of 1,4-cyclohexadiene and the corresponding average for butadiene. ${ }^{41,49} \quad E_{23}=E_{34}(=y)$ is the interaction energy between $\pi\left(\mathrm{CH}_{3}^{(1)}\right)$ and $\pi\left(\mathrm{CH}_{3}^{(2)}\right)$, or $\pi\left(\mathrm{CH}_{3}^{(3)}\right)$ and $\pi\left(\mathrm{CH}_{3}^{(4)}\right)$. It is obtained from the spectra of isobutylene. ${ }^{27}$ The interaction energy between $\pi\left(\mathrm{CH}_{3}\right)$ groups not attached to the same $s p^{2}$ hybridized carbon atom, $E_{24}=E_{25}=E_{34}=E_{35}$, is set equal to zero.

The secular determinantal equation [Eq. (3)] is 
$\left|\begin{array}{ccccc}d-\mu & x & x & x & x \\ x & m-\mu & y & 0 & 0 \\ x & y & m-\mu & 0 & 0 \\ x & 0 & 0 & m-\mu & y \\ x & 0 & 0 & y & m-\mu\end{array}\right|=0$

which has roots $\mu_{i}$

$$
\begin{aligned}
& \mu_{1}=\mu_{2}=m-y \\
& \mu_{3}=m+y \\
& \mu_{4}=\left[d+m+y+\sqrt{(m+y-d)^{2}+16 x^{2}}\right] / 2 \\
& \mu_{5}=\left[d+m+y-\sqrt{(m+y-d)^{2}+16 x^{2}}\right] / 2 .
\end{aligned}
$$

Using $d=10.51 \mathrm{eV}, m=14.2 \mathrm{eV}, x=1.75 \mathrm{eV}$, and $y$ $=0.2 \mathrm{eV}$ yields the eigenvalues for tetramethylethylene in Table VI.

The eigenvector corresponding to $\mu_{i}$ is calculated from the matrix equation

$$
\left(\begin{array}{ccccc}
d-\mu_{i} & x & x & x & x \\
x & m-\mu_{i} & y & 0 & 0 \\
x & y & m-\mu_{i} & 0 & 0 \\
x & 0 & 0 & m-\mu_{i} & y \\
x & 0 & 0 & y & m-\mu_{i}
\end{array}\right)\left(\begin{array}{c}
\mathrm{C}_{1 i} \\
\mathrm{C}_{2 i} \\
\mathrm{C}_{3 i} \\
\mathrm{C}_{4 i} \\
\mathrm{C}_{5 i}
\end{array}\right)=0
$$

and requiring each eigenvector to be normalized. The symmetry designation of each out-of-plane localized orbital is determined from the transformation properties of the eigenvectors relative to reflection and rotation operations of the symmetry group of the molecule being considered.

${ }^{1}$ D. W. Turner, C. Baker, A. D. Baker, and C. R. Brundle, Molecular Photoelectron Spectroscopy (Wiley-Interscience, London, 1970), Chap. 1.

${ }^{2}$ M. I. Al-Joboury and D. W. Turner, J. Chem. Soc. 5141 (1963).

${ }^{3}$ P. Bischof, J. A. Hashmall, E. Heilbronner, and V. Hornung, Helv. Chim. Acta 52, 1745 (1969).

${ }^{4}$ A. D. Baker, D. P. May, and D. W. Turner, J. Chem. Soc. B 22 (1968).

${ }^{5}$ (a) C. R. Brundle and M. B. Robin, J. Am. Chem. Soc. 92, $5550(1970)$; (b) C. R. Brundle, M. B. Robin, and N. A. Kuebler, ibid. 94, 1451 (1972).

${ }^{6}$ F. Wuilleumier and M. O. Krause, Phys. Rev. A 10, 242 (1974).

${ }^{7}$ W. C. Price, A. W. Potts, and D. G. Streets, in Electron Spectroscopy, edited by D. A. Shirley (North-Holland, Amsterdam, 1972), p. 187.

${ }^{8}$ M. B. Robin, N. A. Kuebler, and C. R. Brundle, in Ref. 7, p. 351 .

${ }^{9}$ G. V. Marr and P. R. Woodruff, J. Phys. B 9, L377 (1976).

${ }^{10}$ J. L. Ha11 and M. W. Siegel, J. Chem. Phys. 48, 943 (1968).

${ }^{11} \mathrm{~J}$. Cooper and R. N. Zare, J. Chem. Phys. 48, 942 (1968).

${ }^{12}$ T. A. Carlson and C. P. Anderson, Chem. Phys. Lett. 10, 561 (1971).
${ }^{13}$ T. A. Carison, G. E. MeGuire, A. E. Jonas, K. L. Cheng, C. P. Anderson, C. C. Lu, and B. P. Pullen, in Ref. 7, p. 207.

${ }^{14}$ J. W. Rabalais, T, P. Debies, J. L. Berkosky, J.-T. J. Huang, and F. O. Ellison, J. Chem. Phys. 61, 529 (1974).

${ }^{15}$ D. C. Mason, A. Kuppermann, and D. M. Mintz, in Ref. 7 , p. 269.

${ }^{16}$ R. M. White, T. A. Carlson, and D. P. Spears, J. Electron Spectrosc. 3, 59 (1974).

${ }^{17}$ D. C. Mason, D. M. Mintz, and A. Kuppermann, Rev. Sci. Instrum. 48, 926 (1977); the mean radius and radial gap reported in this reference are in error.

${ }^{18}$ (a) A. D. Baker, C. Baker, C. R. Brundle, and D. W. Turner, Int. J. Mass. Spectrom. Ion Phys. 1, 285 (1968); (b) G. R. Branton, D. C. Frost, T. Makita, C. A. McDowell, and I. A. Stenhouse, J. Chem. Phys. 52, $802(1970)$.

${ }^{19}$ (a) K. Kimura, S. Katsumata, T. Yamazaki, and H. Wakabayashi, J. Electron. Spectrosc. 6, 41 (1975); (b) T. Yamazaki and K. Kimura, Bull. Chem. Soc. Jpn. 48, 1602 (1975); (c) S. Katsumata and K. Kimura, ibid. 49, 442 (1976).

${ }^{20}$ K. B. Wiberg, G. B. Ellison, J. J. Wendoloski, C. R. Brundle, and N. A. Kuebler, J. Am. Chem. Soc. 98, 7179 (1976).

${ }^{21} \mathrm{~J}$. Cooper and R. N. Zare, in Lectures in Theoretical Physics, edited by S. Geltman, K. Mahanthappa, and N. Brittin (Gordon and Breach, New York, 1969), Vol, XI-C, p. 317.

${ }^{22}$ M. I. Al-Joboury and D. W. Turner, J. Chem. Soc. B 4434 (1964).

${ }^{23}$ M. B. Robin, Higher Excited States of Polyatomic Molecules (Academic, New York, 1975), Vol. II, Chap. 4.

${ }^{24}$ M. J. S. Dewar and S. D. Worley, J, Chem. Phys. 50, 654 (1969).

${ }^{25}$ (a) A. W. Potts and D. G. Streets, J. Chem. Soc. Faraday II 70, 875 (1974); (b) D. G. Streets and A. W. Potts, ibid. 70,1505 (1974).

${ }^{26}$ (a) G. G. Hall, Proc. R. Soc. London Ser. A 205, 541 (1951); Trans. Faraday Soc. 49, 113 (1953); (b) J. Lennard-Jones and G. G. Hall, Proc. R. Soc. London Ser. A 213, 102 (1952).

${ }^{27}$ D. C. Frost and J. S. Sandhu, Indian J. Chem. 9, 1105 (1971).

${ }^{28}$ G. Bieri, F. Burger, E. Heilbronner, and J. P. Maier, Helv. Chim. Acta 60, 2213 (1977).

${ }^{29} \mathrm{G}$. Herzberg, Molecular Spectra and Molecular Structure. II. Electronic Spectra and Electronic Structure of Polyatomic Molecules (Van Nostrand, New York, 1967), Chap. II.

${ }^{30}$ D. M. Mintz and A. Kuppermann, J. Chem. Phys. 69, 3953 (1978).

${ }^{31} \mathrm{~J}$. A. Sell, A. Kuppermann, and D. M. Mintz, "Angular distributions in the photoelectron spectroscopy of carbon monoxide," J. Electron Spectrosc. (in press).

${ }^{32} \mathrm{P}$. Natalis and J. E. Collin, Chem. Phys. Lett. 2, 414 (1968); P. Natalis, J. Delwiche, and J. E. Collin, ibid. 13, 491 (1972); J. E. Collin and P. Natalis, Int. J. Mass Spectrom. Ion Phys, 2, 231 (1969).

${ }^{33}$ T. A. Carlson, Chem. Phys. Lett. 9, 23 (1971).

${ }^{34} \mathrm{~J}$. A. Kinsinger and J. W. Taylor, Int. J. Mass Spectrom. Ion Phys. 10, 445 (1972). They used the $58.4 \mathrm{~nm}$ data of Ref. 12 to calibrate their instrument.

${ }^{35}$ B. - Ö. Jonsson and E. Lindholm, Ark. Fys. 39, 65 (1969).

${ }^{36} \mathrm{~W}$. C. Price, in Molecular Spectroscopy, edited by P. Hepple (Institute of Petroleum, London, 1968), p. 221.

${ }^{37}$ H. Bock and B. G. Ramsey, Angew. Chem. Int. Ed. 12, 734 (1973).

${ }^{38}$ R. S. Mulliken, J. Chem. Phys. 7, 339 (1939).

${ }^{39} \mathrm{R}$. Hoffmann, Acc. Chem. Res. 4, 1 (1971).

${ }^{40}$ M. Beez, G. Bieri, H. Bock, and E. Heilbronner, Helv. Chim. Acta 56, 1028 (1973).

${ }^{41}$ T. Koenig and H. Longmaid, J. Org. Chem. 39, 560 (1974) and references cited therein.

${ }^{42}$ The large value of $y(1.2 \mathrm{eV})$ used by $R$. A. Wielesek and 
T. Koenig, Tetrahedron Lett. 28, 2429 (1974), describes the interaction between eclipsed methylene groups and is probably inappropriate for describing the interaction of methyl groups in 2-methyl olefins.

${ }^{43}$ J. - T. J. Huang and F. O. Ellison, Chem. Phys. 7, 473 (1975).

${ }^{44}$ L. L. Lohr, Jr., in Ref. 7, p. 245.
${ }^{45}$ S. T. Manson, J. Electron Spectrosc. 1, 413 (1972).

${ }^{46}$ D. G. McCoy, J. M. Morton, and G. V. Marr, J. Phys. B 11,1547 (1978).

${ }^{47}$ J. L. Dehmer and D. Dill, J. Chem. Phys. 65, 5327 (1977). ${ }^{48}$ E. S. Chang, J. Phys. B 11, L69 (1978).

${ }^{49}$ P. Bishof, J. Hashmall, E. Heilbronner, and V. Hornung, Helv. Chim. Acta 52, 110 (1969). 